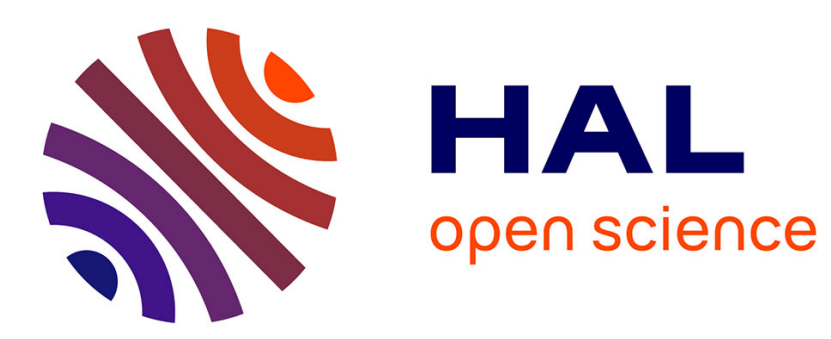

\title{
Fragmentation dynamics of Ar4He1000 upon electron impact ionization: competition between ion ejection and trapping
}

\author{
Nadine Halberstadt, David Bonhommeau
}

\section{> To cite this version:}

Nadine Halberstadt, David Bonhommeau. Fragmentation dynamics of Ar4He1000 upon electron impact ionization: competition between ion ejection and trapping. Journal of Chemical Physics, 2020, 152 (23), pp.234305. 10.1063/5.0009363 . hal-03086651v3

\section{HAL Id: hal-03086651 \\ https://hal.science/hal-03086651v3}

Submitted on 2 Jul 2020

HAL is a multi-disciplinary open access archive for the deposit and dissemination of scientific research documents, whether they are published or not. The documents may come from teaching and research institutions in France or abroad, or from public or private research centers.
L'archive ouverte pluridisciplinaire HAL, est destinée au dépôt et à la diffusion de documents scientifiques de niveau recherche, publiés ou non, émanant des établissements d'enseignement et de recherche français ou étrangers, des laboratoires publics ou privés. 


\section{Fragmentation dynamics of $\mathrm{Ar}_{4} \mathrm{He}_{1000}$ upon electron impact ionization: competition between ion ejection and trapping}

Nadine Halberstadt ${ }^{1}$ and David A. Bonhommeau ${ }^{\text {a) }}$

1) Université Paul Sabatier, CNRS, LCAR UMR 5589, 31062 Toulouse, France.

2) Université de Reims Champagne Ardenne, CNRS, GSMA UMR 7331, 51097 Reims, France.

The fragmentation upon electron impact ionization of $\mathrm{Ar}_{4} \mathrm{He}_{1000}$ is investigated by means of mixed quantum-classical dynamics simulations. The $\mathrm{Ar}_{4}^{+}$dopant dynamics is described by a surface hopping method coupled with a diatomics-in-molecules model to properly take into account the multiple $\mathrm{Ar}_{4}^{+}$electronic surfaces and possible transitions between them. Helium atoms are treated individually using the zeropoint averaged dynamics (ZPAD), a method based on the building of an effective He-He potential. Fast electronic relaxation is observed, from less than 2 ps to $30 \mathrm{ps}$ depending on initial conditions. The main fragments observed $\operatorname{are~} \mathrm{Ar}_{2}^{+} \mathrm{He}_{\mathrm{q}}$ and $\mathrm{Ar}_{3}^{+} \mathrm{He}_{\mathrm{q}}(q \leq 1000)$, with a strong contribution of the bare $\mathrm{Ar}_{2}^{+}$ion, and neither $\mathrm{Ar}^{+}$nor $\mathrm{Ar}^{+} \mathrm{He}_{\mathrm{q}}$ fragments are found. The smaller fragments $(q \leq 50)$ are found to mostly come from ion ejection whereas larger fragments $(q>500)$ originate from long-term ion trapping. Although the structure of the trapped $\mathrm{Ar}_{2}^{+}$ions is the same as in the gas phase, trapped $\mathrm{Ar}_{3}^{+}$and $\mathrm{Ar}_{4}^{+}$are rather slightly bound $\mathrm{Ar}_{2}^{+} \cdots \mathrm{Ar}$ and $\mathrm{Ar}_{2}^{+} \ldots \mathrm{Ar} \cdots \mathrm{Ar}$ structures (i.e., an $\mathrm{Ar}_{2}^{+}$core with one or two argon atoms roaming within the droplet). These loose structures can undergo geminate recombination and release $\mathrm{Ar}_{3}^{+} \mathrm{He}_{q}$ or $\mathrm{Ar}_{4}^{+} \mathrm{He}_{q}(q \leq 50)$ in the gas phase and/or induce strong helium droplet evaporation. Finally, the translational energy of the fragment center of mass was found suitable to provide a clear signature of the broad variety of processes at play in our simulations.

Keywords: mixed quantum-classical dynamics, superfluid helium nanodroplets, ionicrare-gas clusters, ionization induced fragmentation, zero-point energy, ejection, trapping.

a) Author to whom correspondence should be addressed. Electronic mail: david.bonhommeau@univ-reims.fr 


\section{INTRODUCTION}

At the very low temperature of their formation, $T \approx 0.37 \mathrm{~K},{ }^{1}$ superfluid helium nanodroplets have revealed to be an ideal environment to perform high resolution spectroscopy, ${ }^{2,3}$ to form molecular assemblies that would be unstable in the gas phase, ${ }^{4}$ or to grow metallic nanocluster wires which can be deposited on surfaces for applications in optics or catalysis. ${ }^{5}$ These applications are based on the helium superfluid properties at low temperature (no viscosity, no entropy). However, the property of frictionless motion is only valid at low energy excitations. Upon ionization of a doped droplet, the electronic hole initially created on a helium atom is prone to migrate to the neutral dopant, the efficiency of this charge transfer depending on the droplet size and dopant nature. ${ }^{6-11}$ The ensuing dopant ionization is likely to make it dissociate, a process which is well known for rare-gas clusters where the large equilibrium structure difference between the neutral cluster and the parent ion, as well as the availability of multiple excited electronic states of the latter, can bring a lot of internal energy into the process. ${ }^{12-20}$ In this case, the speed of the fragmenting dopant atoms usually exceeds the Landau critical velocity, $v_{L} \approx 55-60 \mathrm{~m} \mathrm{~s}^{-1}$ at $T=1 \mathrm{~K}$ and $p=1 \mathrm{~atm} .{ }^{21}$ These atoms thus undergo a friction force due to the helium environment. If their velocity is not too large they are trapped inside the droplet which can lead to geminate recombination ${ }^{22}$ or "caging".

The study of the cage effect exerted by helium atoms on ionized dopants has attracted a lot of interest during the past decades. For instance, electron impact ionization of $\mathrm{SF}_{6}$ in a helium droplet only yields $\mathrm{SF}_{6}^{+}$and $\mathrm{SF}_{5}^{+}$whereas $\mathrm{F}^{+}$and all the $\mathrm{SF}_{n}^{+}(n=0-5)$ fragments are produced upon gas phase ionization at $T=300 \mathrm{~K} .{ }^{7,23}$ This result evidenced a significant hindering of the fragmentation by the surrounding helium atoms. This caging is even more efficient for $\mathrm{NO}_{2}^{+}$whose fragments remain in the droplet and are therefore prone to recombine before detection. ${ }^{24}$ Assuming thermal evaporation, the ejection of a single helium atom from large nanodroplets is expected to dissipate about $5 \mathrm{~cm}^{-1}$ of energy, which should provide an easy way of assessing the cooling power of a helium droplet. ${ }^{6,25,26}$ However, Lewis et al. ${ }^{27}$ found that the first 5000 helium atoms removed about $22 \mathrm{~cm}^{-1} /$ atom in the case of triphenylmethanol (TPM) electron impact ionization, although the cooling from 10000 to 40 000 helium atoms only removed $0.16 \mathrm{~cm}^{-1} /$ atom. A similar nonthermal evaporation has been observed for HCN molecules whose fragmentation could be accurately measured by coupling 
helium droplet mass spectrometry and infrared laser spectroscopy. ${ }^{28}$ More recently, Braun et al. investigated the translational dynamics of alkyl iodides photodissociated in helium nanodroplets. ${ }^{29,30}$ Besides the expected influence of droplet size on caging, they noticed that $\mathrm{CF}_{3}$ and $\mathrm{C}_{2} \mathrm{H}_{5}$ photofragments escaped the helium droplets by nonthermal direct processes with speed distributions properly modeled by assuming independent binary hard-sphere collisions between the fragments and the surrounding helium atoms. On the contrary, $\mathrm{CH}_{3}^{+}$ fragments were only detected for small photoexcited droplets in velocity map images, which lead them to conclude that the $\mathrm{CH}_{3}$ and I photofragments were subject to recombinations. ${ }^{31}$

The significant caging by helium atoms observed experimentally is in stark contrast with the theoretical results obtained by Takayanagi et al. for the photodissociation of $\mathrm{Cl}_{2}$ in $\mathrm{He}_{200} \cdot{ }^{32}$ These authors took into account quantum effects in their model by combining quantum wavepacket dynamics for $\mathrm{Cl}_{2}$ and path integral centroid molecular dynamics for helium atoms. Dissociation of $\mathrm{Cl}_{2}$ occurred in almost $100 \%$ of the trajectories. In a more recent study combining He-TDDFT for the helium density and a wave packet description of the $\mathrm{Cl}-\mathrm{Cl}$ coordinate, Vilá et al. ${ }^{33}$ also observed complete dissociation of $\mathrm{Cl}_{2}$ and escape of $\mathrm{Cl}$ fragments from a droplet of up to $500{ }^{4} \mathrm{He}$ atoms, together with the ejection of only up to $10{ }^{4} \mathrm{He}$ atoms within the first 3 ps (about 50 atoms after $150 \mathrm{ps}$ ) 34 , even though $91 \%$ of the diatomic energy was released in the droplet. Interesting quantum interferences in the velocity distributions were assigned to the effect of the surrounding helium environment. ${ }^{35}$

We have previously studied the fragmentation dynamics upon electron impact ionization of small neon-doped helium clusters, $\mathrm{Ne}_{n}^{+} \mathrm{He}_{100}(n=4-6)$, using a mixed quantum-classical $\operatorname{method}^{36}$ where the nonadiabatic dynamics of the central neon cluster was modeled by surface hopping techniques while helium atoms moved classically on an effective potentialenergy surface (PES) including average zero-point effects (ZPAD method). ${ }^{37,38}$ The results showed that $\mathrm{Ne}_{n}^{+}$fragmentation was mostly completed within the first 30 ps. By comparing with gas phase ionization, the fragment distributions revealed some caging (Table IX in Ref. 38) although in a minor proportion. For instance, fewer fragments contained $\mathrm{Ne}^{+}$ (2.6\% instead of $11.2 \%$, for $\mathrm{Ne}_{4}$ ionization in $\mathrm{He}_{100}$ and in gas phase, respectively), and some $\mathrm{Ne}_{3}^{+}$-containing fragments were observed (10.5\%) subsequent to $\mathrm{Ne}_{6}$ ionization in $\mathrm{He}_{100}$ rather than traces $(0.1 \%)$ in gas phase. In addition, this study evidenced a dopant ejection mechanism as well as nonthermal evaporation of helium atoms, with $28-45 \mathrm{~cm}^{-1} /$ atom lost on average depending on the cluster size. Finally, $\mathrm{Ne}_{p}^{+}(2 \leq p \leq n)$ and $\mathrm{Ne}_{p}^{+} \mathrm{He}_{q}$ 
( $1 \leq p \leq n, q \leq 100)$ fragment abundances provided valuable data sets to be compared with experiments. $^{39-42}$

In both the study by Takayanagi et al. ${ }^{32}$ and ours, ${ }^{37,38}$ the modest size of the droplet compared to typical experimental sizes could explain the absence or near absence of caging. Even the 500-atom droplet seemed too small to observe any caging in the study by Vilá et $a l .{ }^{33}$ In the present work, we extend our former study of neon-doped helium clusters to larger argon-doped helium clusters, considering more specifically $\mathrm{Ar}_{4}^{+} \mathrm{He}_{1000}$ as a prototype system for large helium droplets. Argon clusters are preferred to neon or heavier rare gases (i.e., krypton or xenon) clusters due to a number of properties: a more classical character compared to neon, that is a low zero-point energy and delocalization; moderate spin-orbit coupling; ${ }^{43}$ the high natural abundance of isotope ${ }^{40} \mathrm{Ar}(\sim 99.6 \%)$ which makes it easier to compare with experimental mass spectra; ${ }^{44}$ and the availability of both theoretical and experimental benchmark data on the fragmentation of argon clusters ionized by electron impact in the gas phase. ${ }^{15,19,20,45,46}$

On the experimental side, Callicoatt et al. ${ }^{9}$ have investigated the fragmentation of argondoped helium nanodroplets upon electron impact ionization. They mostly detected fragments composed of up to four argon atoms after ionizing their smaller helium nanodroplets (droplet size $\sim 600-8000$ ) with $60 \mathrm{eV}$ electrons. They concluded that singly-doped droplets yielded $\mathrm{Ar}^{+} \mathrm{He}_{q}$ but no $\mathrm{Ar}^{+}, \mathrm{Ar}_{2}$ doped droplets mainly yielded $\mathrm{Ar}_{2}^{+}$, and $\mathrm{Ar}_{3}$ and $\mathrm{Ar}_{4}$-doped droplets mostly gave $\mathrm{Ar}_{2}^{+}$. Upon photoionization of argon-doped helium droplets, Kim et al. ${ }^{11}$ have observed $\mathrm{Ar}^{+} \mathrm{He}_{q}, \mathrm{Ar}_{2}^{+}$and $\mathrm{Ar}_{2}^{+} \mathrm{He}_{q}$, but no $\mathrm{Ar}^{+}$.

More recently, Bartl et al. investigated the fragmentation of argon-doped helium nanodroplets (droplet size $\sim 10^{4}-10^{6}$ ) ionized by electrons with energies ranging from 70 to $140 \mathrm{eV} .{ }^{42}$ Thanks to the high resolving power of their mass spectrometer $(m / \Delta m=7000)$, they could easily discriminate between $\mathrm{Ar}_{n}^{+} \mathrm{He}_{q}$ fragments with nominally identical masses (the mass of an argon atom is close to that of ten helium atoms). They reported progressions of $\mathrm{Ar}_{n}^{+} \mathrm{He}_{q}$ fragments for $n \leq 7$ and $q \leq 50$. They observed that bare cluster ions $\mathrm{Ar}_{n}^{+}$ and mixed complexes $\mathrm{Ar}_{n}^{+} \mathrm{He}_{q}$ had about the same yield $(n>1)$. They also discussed the origin of about twenty magic numbers from the smaller $\mathrm{Ar}^{+} \mathrm{He}_{12}$ to the larger $\mathrm{Ar}_{7}^{+} \mathrm{He}_{12}$, and noted an enhanced stability for all the clusters with $n+q=19(2 \leq n \leq 7)$. Although the theoretical check of magic numbers would be a significant challenge for dynamics simulations, owing to the experimental width of droplet and dopant size distributions as well 
as the great number of lengthy trajectories required to converge theoretical mass spectra intensities, some of the observables, e.g. the proportion of bare cluster ions, might be related to the electronic relaxation of the excited dopant, the subsequent energy distribution within the droplet, and possible recombinations, three aspects of the fragmentation mechanisms of argon-doped helium droplets that can be investigated with our mixed quantum-classical method.

We will first recall in the Method section some essential features of the mixed quantumclassical method and detail the improvements brought to model the dynamics of $\mathrm{Ar}_{4}^{+} \mathrm{He}_{1000}$. A brief discussion of the influence of the model parameters (effective He-He interaction potentials and velocity adjustment techniques) is reported in the Supplementary Material. The Results section focuses on the fast character of electronic relaxation, ionic fragment abundances, characteristic times of the dynamics, and proposes two extreme mechanisms, fast ejections and long-term trappings, before studying the possibility to use fragment kinetic energy distributions to characterize them. The final section discusses the results and concludes in the perspective of a more extensive study.

\section{METHOD}

Ionization-induced fragmentation of $\mathrm{Ar}_{4}$ clusters embedded in a helium nanodroplet of 1000 atoms has been simulated using a method similar to that employed in our previous work on the fragmentation of $\mathrm{Ne}_{n}(n=4-6)$ clusters upon electron impact ionization in a ${ }^{4} \mathrm{He}_{100}$ droplet. ${ }^{37,38}$ We recall here the essential features of the original method and stress the modifications brought in the current work.

This study faces the double challenge of modelling the multisurface dynamics of the ionized argon cluster and the significant quantum effects due to a large number of helium atoms. So far, only the time-dependent ${ }^{4} \mathrm{He}$ density functional method $\left({ }^{4} \mathrm{He}-\mathrm{TDDFT}\right)$ has been able to accurately describe excited state dynamics for a realistic droplet size. ${ }^{47,48}$ However, the electronic state of the dopants is described in a mean-field approach, which does not identify electronic transitions. Ring polymer molecular dynamics (RPMD) has been tested, initially on potassium ${ }^{49}$ or silver ${ }^{50}$ atom excitation and $\mathrm{Cl}_{2}$ photodissociation ${ }^{32}$ in small helium droplets, and more recently to investigate size-dependent submersion of sodium clusters into helium and para-hydrogen droplets. ${ }^{51}$ These studies involved only one electronic 
state and did not take into account ${ }^{4} \mathrm{He}$ atom exchange. We have preferred to use a more approximate method, in order to explicitly track electronic state transitions and identify individual helium atoms, at the expense of a more approximate description of quantum effects: zero-point delocalization is treated phenomenologically using the ZPAD method, while exchange-correlation effects are neglected. At the high energy of the dynamics induced by argon clusters ionization, it is expected to be a reasonable approximation.

\section{A. Essential features of the ZPAD method}

In order to take into account, at least approximately, the quantum delocalization of the helium atoms, the ZPAD method is based on the idea of "dressing" each helium atom with a wave function that will be kept frozen during the dynamics. This is the same idea as the frozen-Gaussian approach ${ }^{52}$ adapted by Sterling et al. ${ }^{53}$ to study the dynamics of dopants in quantum crystals. The version used here is based on the method described by Slavíček et al. ${ }^{54}$ to describe the photodissociation of $\mathrm{HBr}$ and $\mathrm{HCl}$ in floppy neon clusters. It has the advantage that the wave function dressing each helium atom is not restricted to be a Gaussian, and that the method is iterative and self-consistent. It has been adapted for helium atoms in Refs. 37 and 38.

As in the frozen-Gaussian approach, using a frozen wave function during the dynamics is equivalent to running classical dynamics on an effective potential, which is the original potential convolved with the squared wave function. Hence, the method is able to treat a large number of helium atoms. Both the wave function and the effective potential are determined at the same time by an iterative procedure. In the preliminary step, a classical dynamics is run for the neutral $\mathrm{Ar}_{4} \mathrm{He}_{1000}$ cluster at the droplet temperature $(0.38 \mathrm{~K}){ }^{55}$ The potential-energy surface is built from the HFDID1 Ar-Ar interaction potential, ${ }^{56}$ the HFD-B He-Ar interaction potential,,$^{57,58}$ and the SAPT2 He-He potential proposed by Janzen and Aziz, ${ }^{59}$ namely

$$
V_{\text {neut }}(\mathbf{R})=\sum_{i=1}^{4} \sum_{\substack{j=1 \\ j>i}}^{4} V_{\mathrm{Ar}-\mathrm{Ar}}\left(R_{i j}\right)+\sum_{i=1}^{4} \sum_{a=1}^{1000} V_{\mathrm{He}-\mathrm{Ar}}\left(R_{i a}\right)+\sum_{a=1}^{1000} \sum_{\substack{b=1 \\ b>a}}^{1000} V_{\mathrm{He}-\mathrm{He}}\left(R_{a b}\right)
$$

where $\mathbf{R}$ stands for all the internuclear distances. The corresponding Ar-Ar, He-Ar, and He-He interaction potentials are depicted in Figure 1. 
Then, the following procedure is applied:

1. The classical radial distribution function (rdf) of helium atoms, normalized by assuming that the cluster is spherical, is built on the fly during the dynamical run of step $i$, using the effective He-He potential of step $i-1$. For $i>1$, it is subsequently convolved with helium wavefunction of step $i-1$ to produce a semiclassical rdf.

2. The semiclassical rdf is convolved with the SAPT2 He-He potential to provide the average radial potential felt by one helium atom in the mean field due to the others.

3. This average radial potential is included in a Schrödinger equation, the solution of which gives the helium wave function for step $i$.

4. The original He-He interaction potential is convolved with the newly calculated helium wavefunction to build the effective He-He potential for step $i$.

This procedure is initiated with the original SAPT2 He-He potential. ${ }^{59}$ Steps (1) to (4) are repeated until convergence of the effective potential. In previous works ${ }^{37,38}$ we found it practical to remove the tail of the helium wave function beyond a cutoff radius $R_{\text {cut }} \approx$ $1.5-1.6 \AA$ in order to reach convergence more efficiently. This was not necessary in the original study of Slavíček et al. ${ }^{54}$ for neon clusters since the effective Ne-Ne potential curves converged after as few as 4 iterations, to be compared with the 27 iterations needed to converge the effective He-He potential curve for $R_{\text {cut }}=1.6 \AA$ in the present work. For this reason, we expect our converged effective He-He potential to be slightly too deep to represent liquid helium droplets since the well depth of the effective potential decreases with larger cutoff radii. However, the helium radial probability density functions deduced from such potentials in the case of neon-doped helium clusters were found to compare very well with diffusion Monte Carlo (DMC) simulations, ${ }^{37}$ which makes us believe that most of the quantum effects are indeed taken into account. In the following, the ZPAD method will refer to both the iterative process that aims to incorporate zero-point energy effects in the He-He interaction potential (potential part of the ZPAD method) and the subsequent dynamics of helium atoms performed using this effective potential (dynamical part of the ZPAD method). 


\section{B. Effective potential, structure and energy of $\mathrm{Ar}_{4} \mathrm{He}_{1000}$}

The final effective He-He potential is reported in Figure 1, together with the ones obtained for several iterations, to highlight the convergence of the aforementioned iterative procedure for the potential part of the ZPAD method. In order to validate it, the surface tension of $\mathrm{Ar}_{4} \mathrm{He}_{1000}$ clusters was calculated and compared with the experimental data from Iino et al. ${ }^{26}\left(\gamma_{\exp }=3.536 \times 10^{-4} \mathrm{~N} / \mathrm{m}\right)$ using the approximate formula for homogeneous clusters of radius $R$ and size $N$

$$
\gamma_{t h}=\frac{E_{s}}{4 \pi R^{2}}=\frac{\left(A+B N^{-1 / 3}\right) V_{L J}}{(36 \pi)^{1 / 3} \sigma_{L J}^{2}}
$$

where the expression of surface energy $E_{s}=\left(A N^{2 / 3}+B N^{1 / 3}\right) V_{L J}(A=10.3$ and $B=$ -2.7 are two dimensionless constants and $V_{L J}$ is the corresponding Lennard-Jones (LJ) pair well depth) has been devised by Echt et al. ${ }^{60}$ on the basis of the work by Briant and Burton on argon microclusters, ${ }^{61}$ and the approximate radius of the cluster is given by $R=(3 N / 4 \pi)^{1 / 3} \sigma_{L J}$, with $\sigma_{L J}$ the pair diameter of LJ particles (helium atoms here). For the effective He-He potential with $R_{\text {cut }}=1.6 \AA, V_{L J}=1.254 \mathrm{~cm}^{-1}\left(2.491 \times 10^{-23} \mathrm{~J}\right)$ and $\sigma_{L J}=R_{e q} / 2^{1 / 6}=3.92 \AA\left(R_{e q}=4.40 \AA\right.$ is the equilibrium He-He distance), and we get $\gamma_{t h}=3.362 \times 10^{-4} \mathrm{~N} / \mathrm{m}$ which is $5 \%$ below the bulk surface tension at $T=0.4 \mathrm{~K}$. This is a reasonable agreement although the stabilization of the droplet by the dopant makes He-He bonds somewhat shorter and the He-He potential somewhat deeper than those expected for pure helium nanodroplets.

In order to reach a better accuracy in the classical dynamics, the resulting ZPAD effective potential points were fitted to an analytical form

$$
V_{\mathrm{He}-\mathrm{He}}^{\mathrm{eff}}(R)=V_{\text {short }}(R)+T(R)\left[V_{\text {long }}(R)-V_{\text {short }}(R)\right]
$$

where

$$
\begin{aligned}
V_{\text {short }} & =A \exp \left[-\alpha_{1} R / R_{e}-\alpha_{2}\left(R / R_{e}\right)^{2}\right] \\
V_{\text {long }} & =\sum_{i=3}^{6} c_{2 i}\left(R / R_{e}\right)^{-2 i} \\
T(R) & =0.5(1+\tanh [a(R-b)]) .
\end{aligned}
$$

This expression slightly improves the fit in the short-range region of the potential curve of previous studies by adding a quadratic term $\alpha^{2}\left(R / R_{e}\right)^{2}$ in the exponential argument, ${ }^{37,38,62}$ 
at the expense of additional computational cost. The fit parameters obtained for $R_{\text {cut }}=$ $1.6 \AA$ are collected in Table I. Note that parameter $R_{e} \approx 4.43 \AA$, obtained by fitting the bottom of the effective He-He potential well on a harmonic oscillator and subsequently used to initiate the overall fitting process of the effective potential, slightly differs from the true equilibrium distance of the analytical effective He-He potential $\left(R_{e q} \approx 4.40 \AA\right)$ considered to evaluate the droplet surface tension.

After converging the effective He-He potential, a last dynamical run is carried out to get an $\mathrm{Ar}_{4} \mathrm{He}_{1000}$ structure equilibrated at $T=0.38 \mathrm{~K}$. This structure is used as input configuration in our mixed quantum-classical method devised to model the fragmentation of $\mathrm{Ar}_{4}^{+} \mathrm{He}_{1000}$.

\section{Mixed quantum-classical dynamics of $\mathrm{Ar}_{4}^{+} \mathbf{H e}_{1000}$}

The dynamical simulations are divided into three successive steps: an equilibration of the neutral cluster prior to its ionization by electron impact, a nonadiabatic dynamics of the ionized cluster during which electronic transitions can take place, and a long-term adiabatic fragmentation dynamics once electronic relaxation to the ground electronic state is completed.

The first step consists in running a ZPAD trajectory for the sake of equilibrating the neutral $\mathrm{Ar}_{4} \mathrm{He}_{1000}$ cluster. As explained above, ZPAD is equivalent to classical dynamics using the effective He-He interaction obtained as described in the preceding section. Hence, the potential-energy surface is that reported in Eq.(1), except that the SAPT2 He-He interaction potential $V_{\mathrm{He}-\mathrm{He}}(R)$ is replaced by the converged effective He-He potential $V_{\mathrm{He}-\mathrm{He}}^{\text {eff }}(R)$ from Eq.(3). This equilibration dynamics starts from the $\mathrm{Ar}_{4} \mathrm{He}_{1000}$ positions and velocities obtained upon convergence of the He-He effective potential, namely one particular phasespace configuration corresponding to a kinetic temperature of $0.38 \mathrm{~K}$. Although this initial configuration is the same for all the trajectories, the random duration of the ZPAD trajectory ensures a reasonable exploration of $\mathrm{Ar}_{4} \mathrm{He}_{1000}$ phase space and therefore the production of distinct configurations used as input for the dynamics of the ion.

The second step is the simulation of the dynamics following dopant ionization. The most likely first event in electron impact ionization of a doped helium cluster is the creation of a positive $\mathrm{He}^{+}$hole within the droplet (see for instance Ref. 9). It is then followed 
by charge migration inside the droplet, until either $\mathrm{Ar}_{4}$ is ionized by charge transfer from $\mathrm{He}^{+}$or the charge is localized to form $\mathrm{He}_{2}^{+}$. In order to specify accurate initial conditions for the electronic wave packet and to select the initial potential-energy surface on which the dynamics starts, the whole process would have to be simulated, which is beyond the scope of this work. Hence, in the absence of any other indications, we have assumed "instantaneous" $\mathrm{Ar}_{4}$ ionization. In other words, at the end of each equilibrated trajectory described above, the whole system is switched to the ionic state by randomly selecting one of the adiabatic surfaces of $\mathrm{Ar}_{4}^{+}$converging to $\mathrm{Ar}^{+}\left({ }^{2} \mathrm{P}\right)+3 \mathrm{Ar}$, denoted $V_{j}$ for $j \in[1,12]$. The random selection of the initial electronic state is reflected in the electronic wave packet by taking identical initial weights for all electronic states. Note that all the (spin-free) electronic states correlating with ground state $\mathrm{Ar}^{+}\left({ }^{2} \mathrm{P}\right)+3 \mathrm{Ar}$ are considered in this study. Electronic states of $\mathrm{Ar}_{4}^{+}$correlating to electronically excited $\mathrm{Ar}^{+}$are not included since they cannot be reached by charge transfer.

From there on, the second step in our simulations is mixed quantum-classical nonadiabatic dynamics of the ionized cluster, in which the argon and helium nuclei are treated classically but the electronic hole, only delocalized on argon atoms, is treated quantum mechanically. We use the molecular dynamics with quantum transitions (MDQT) method. ${ }^{63,64}$ The classical dynamics of the nuclei is run on one adiabatic surface, and the multisurface character of the simulation is taken into account by allowing for hops between $\mathrm{Ar}_{4}^{+}$adiabatic surfaces. The adiabatic energies are obtained by diagonalizing the diatomics-in-molecules (DIM) model hamiltonian ${ }^{65-67}$ for $\mathrm{Ar}_{4}^{+}$in the basis set of the twelve argon effective $p$ orbitals. The matrix elements are constructed from the analytical expressions of the Ar-Ar and $\mathrm{Ar}_{2}^{+}$ diatomic potentials, ${ }^{19,56}$ supplemented by the addition of the induced dipole-induced dipole interaction, with electric dipole polarizability $\alpha=1.664 \AA^{3}{ }^{68}$ The total potential-energy surface is then built from the relevant $\mathrm{Ar}_{4}^{+}$adiabatic electronic state $V_{j}$, an average $\mathrm{He}-\mathrm{Ar}^{+}$ interaction potential, denoted $\bar{V}_{\mathrm{He}-\mathrm{Ar}^{+}}$, and the same He-Ar and effective He-He interaction potentials as used for the dynamics of the neutral cluster,

$$
\begin{aligned}
V_{\mathrm{ion}}^{j}(\mathbf{R}) & =V_{j}+V_{\mathrm{He}-\text { dopant }}^{j}+\sum_{a=1}^{1000} \sum_{\substack{b=1 \\
b>a}}^{1000} V_{\mathrm{He}-\mathrm{He}}^{\mathrm{eff}}\left(R_{a b}\right) \\
V_{\mathrm{He}-\text { dopant }}^{j} & =\sum_{i=1}^{4} \sum_{a=1}^{1000}\left[q_{i}^{(j)} \bar{V}_{\mathrm{He}-\mathrm{Ar}^{+}}\left(R_{i a}\right)+\left(1-q_{i}^{(j)}\right) V_{\mathrm{He}-\mathrm{Ar}}\left(R_{i a}\right)\right]
\end{aligned}
$$

where $\mathbf{R}$ stands for all the internuclear distances, $V_{j}$ is the $j^{\text {th }}$ adiabatic energy of $\mathrm{Ar}_{4}^{+}, q_{i}^{(j)}$ is 
the fractional charge carried by the argon atom $i$ (see Appendix) for that adiabatic energy, and the average $\mathrm{He}-\mathrm{Ar}^{+}$potential is built as a weighted sum of the analytical forms proposed by Carrington et al. ${ }^{69}$ for the $\mathrm{X}^{2} \Sigma^{+}$and $\mathrm{A}^{2} \Pi$ electronic states of $\mathrm{He}^{-\mathrm{Ar}^{+}}$(see Figure 1 ),

$$
\bar{V}_{\mathrm{He}-\mathrm{Ar}^{+}}\left(R_{i a}\right)=\frac{1}{3} V_{\mathrm{He}-\mathrm{Ar}^{+}}^{X^{2} \Sigma^{+}}\left(R_{i a}\right)+\frac{2}{3} V_{\mathrm{He}-\mathrm{Ar}^{+}}^{A^{2} \Pi}\left(R_{i a}\right) .
$$

Note that the spin-orbit interaction is not taken into account in order to keep the simulations within reasonable computational cost. As commented in our previous work on gas phase ionization of $\mathrm{Ar}_{n}$ clusters, ${ }^{19,20}$ the influence of spin-orbit coupling on ionization induced fragmentation was negligible for cluster sizes greater than $n=6$, and it was already very small for $n=4$ and 5 .

In our implementation of the DIM+MDQT method ${ }^{70}$ the probability for the system to hop from an adiabatic surface $j$ to an adiabatic surface $k$ is given by

$$
P_{j \rightarrow k}=-2 \Delta t \frac{\xi_{k}}{\xi_{j}} \dot{\mathbf{R}} \cdot \mathbf{d}_{k j} \cos \left(\varphi_{k}-\varphi_{j}\right)
$$

where $\Delta t$ is the current time step of the dynamics, $\xi_{j}$ and $\varphi_{j}$ are the modulus and the phase of the $j^{\text {th }}$ time-dependent wave packet coefficient (corresponding to the time-evolution of the positive charge), and $d_{k j}$ is the nonadiabatic coupling vector between surfaces $k$ and $j$ calculated from the Hellmann-Feynman formula. If a hop from surface $j$ to surface $k$ is accepted, the total kinetic energy has to be adjusted in order to ensure total energy conservation. In the original MDQT method this is done by adjusting atomic momenta along the nonadiabatic coupling vector $\mathbf{d}_{k j} \cdot{ }^{63,64}$ However, this choice does not conserve the total angular momentum when applied to model the fragmentation dynamics of ionized neon clusters in the gas phase. ${ }^{17}$ We had chosen instead the direction of the gradient of the energy difference between adiabatic states $k$ and $j, g_{k j}=\nabla\left(V_{k}-V_{j}\right) .{ }^{71-73}$ In the present work, where helium atoms are explicitly taken into account in the dynamics, the partial charge $q_{i}^{(j)}$ on argon atom $i$ depends on the electronic state $j$. Hence, upon a hop, the potential is also suddenly modified for the helium atoms dynamics, as seen in the second term of the right-hand side of Eq.(5). We have therefore chosen the gradient $g_{\mathrm{He}}=\nabla\left(V_{\mathrm{He}-\text { dopant }}^{k}-V_{\mathrm{He}-\text { dopant }}^{j}\right)$, where $V_{\mathrm{He} \text {-dopant }}^{j}$ is the He-dopant interaction calculated for charges representative of adiabatic state $j$ and defined in Eq.(6), to adjust the argon and the helium momenta. Very similar results are obtained when using $g_{k j}$ as discussed in the Supplementary Material.

The third step of the mixed quantum-classical simulation is an adiabatic classical run 
performed for trajectories that reached the ground electronic state ( $>95 \%$ of the trajectories) at the end of the $100 \mathrm{ps}$ of the second step (i.e., the nonadiabatic step). Nuclei evolve adiabatically on the ground ionic electronic surface, i.e. the ionic surface of Eq.(5) where $V_{j}$ is the ground electronic state of $\mathrm{Ar}_{4}^{+}$. This adiabatic simulation is devoted to the study of possible fragmentation and recombination mechanisms that might occur on longer time scales, up to $0.5 \mathrm{~ns}$ in this work.

\section{Computational details}

A typical dynamical simulation is a set of 160 trajectories where the classical positions and momenta and the wave packet coefficients (modulus and phase) are propagated using the Hamming's predictor corrector integrator. ${ }^{74}$ The initial simulation time step and duration were $\Delta t_{1}=0.1$ fs and $t_{1}=10(1+\xi)$ ps for initial randomization in the neutral cluster, with $\xi$ a random number between 0 and $1 ; \Delta t_{2}=0.1 \mathrm{fs}$ and $t_{2}=100 \mathrm{ps}$ for the nonadiabatic dynamics of the ionic cluster; $\Delta t_{3}=1 \mathrm{fs}$ and $t_{3}=400 \mathrm{ps}$ for the final adiabatic dynamics. The justification of this choice for $t_{2}$ and $t_{3}$ is given in the Results section. Data are stored every 0.1 ps during the nonadiabatic run and every 1 ps during the adiabatic run.

In both nonadiabatic and adiabatic runs, the integration of the classical equations of motion is very accurate because the potential derivatives of $V_{\text {neut }}(\mathbf{R})$ and $V_{\text {ion }}(\mathbf{R})$ are known analytically. Only the derivatives of the charges $q_{i}^{(j)}$ are numerical (see Appendix). Furthermore, many fragmentation and recombination processes occur during the simulations. Individual fragments are identified by applying a distance criterion $d_{\text {frag }}=15$ au $(\sim 8 \AA)$ to decide whether a bond is broken or not, and taking care that all the atoms belonging to a fragment are at least at $d_{\text {frag }}$ from any of the atoms belonging to other fragments. ${ }^{75}$ We have checked that the resulting fragment distributions are only barely sensitive to the value of $d_{\text {frag }}$.

\section{RESULTS}

\section{A. Electronic relaxation of the $\mathrm{Ar}_{4}^{+}$parent ion}

The average relaxation time of $\mathrm{Ar}_{4}^{+}$to its ground electronic state (whether fragmented or not) is plotted in Figure 2 as a function of the initial excited electronic state. For most 
states (the 8 lowest ones), relaxation to the ground electronic state is faster than 2 ps. This relaxation time increases with excited state energy, and electronic relaxation takes somewhat longer when the dynamics starts on one of the upper states. However, it does not exceed a few tens of picoseconds on average. These observations validate our two-step simulation composed of a nonadiabatic simulation during 100 ps, to allow for electronic relaxation, followed by an adiabatic simulation on the ground electronic state up to 500 ps.

The average initial energy of the electronic states involved is also depicted as a function of electronic state number as an inset in Figure 2. There is a relationship between the electronic state initial energy and its relaxation time, in the sense that higher electronic states have more available energy to dissipate, which on average takes longer. However, the relationship is not simple, as the dispersion of relaxation times is also highest for these excited states. For instance, the relaxation times for state 12 range from about 2 ps to 77 ps. This is because electronic relaxation rates strongly depend not only on the static nonadiabatic couplings, but also on velocity vectors in the regions of strong couplings (see Eq. (8)). In addition, the number of intermediate states visited by a trajectory also depends on the dynamics.

\section{B. Ionic fragment abundances}

A number of ionic species are formed during the fragmentation of the ionized doped helium droplet. To clarify the discussions on fragmentation mechanisms and fragment abundances below, the term "dopant" will refer to all the host argon atoms inside the (initial or shrunk) helium droplet, whether ionic or not. "Ionic fragments" will designate the most general ionic fragments containing argon and helium atoms, namely $\operatorname{Ar}_{n}^{+} \mathrm{He}_{q}(2 \leq n \leq 4, q \leq 1000)$ fragments reported in theoretical mass spectra. "Ionic core" will only refer to the compact charged argon cluster within an ionic fragment, since no helium atom can carry a charge in our simulation, which is a very good approximation given the difference in ionization potential between Ar and He. A "roaming" argon atom will designate a neutral argon atom, whether dissociated from the ionic dopant or barely bound to it, which moves inside the droplet.

Figure 3 shows the mass spectra obtained at the end of the 100 ps nonadiabatic simulation step, during which electronic relaxation can occur, and at the end of the overall 500 ps simulation. Two main results emerge form their analysis: the absence of $\mathrm{Ar}^{+}$and $\mathrm{Ar}^{+} \mathrm{He}_{q}$ 
fragments, and the production of two families of ionic fragments. The first family gathers small $\mathrm{Ar}_{2}^{+} \mathrm{He}_{q}$ and $\mathrm{Ar}_{3}^{+} \mathrm{He}_{q}(q \leq 50)$ ions with a significant preference for the $\mathrm{Ar}_{2}^{+}$core and a cloud of helium atoms often below twenty atoms. It mainly appears as a relatively narrow set of peaks extending from 80 to $140 \mathrm{amu}$. The second family gathers large ions that can contain up to four argon atoms. It is represented by a broad distribution of peaks extending from a few hundreds amu to more than three fourth of the initial droplet mass and gradually shifts to lower masses between 100 ps and 500 ps.

The absence of $\mathrm{Ar}^{+}$and $\mathrm{Ar}^{+} \mathrm{He}_{q}$ fragments appears to be in stark contradiction with the intense signal observed for $\mathrm{Ar}^{+}$and $\mathrm{Ar}^{+} \mathrm{He}_{q}$ in experimental mass spectra by Bartl et al. ${ }^{44}$ However, argon clusters are not size selected in these experiments, so the $\mathrm{Ar}^{+}$containing ions could originate from $\mathrm{Ar}_{n}$ dopants with $n<4$. Indeed, in previous simulations on gas phase argon cluster ionization, ${ }^{19}$ the ionic monomer fragment was observed for small parent sizes only. Its abundance was $9.1 \pm 0.4 \%$ for $\mathrm{Ar}_{4}$ with no inclusion of spin-orbit coupling and $13.8 \pm 0.6 \%$ when this interaction was taken into account. Hence, the intense monomer peak observed in experiments most probably originates from cluster sizes smaller than the tetramer studied here. Other explanations could be the occurrence of double ionization $^{9,76}$ or possible secondary ionization of neutral Ar fragments. ${ }^{19}$ Radiative relaxation on a longer (microsecond) time scale explored for heavier rare gases by Janeček et al. ${ }^{77}$ would not apply here since electronic relaxation is complete at the end of the nonadiabatic step of the simulation.

More details on the ionic fragment abundances are given in Figure 4. As can be seen in this figure, the most abundant "bare" (i.e. with no helium attached) argon ion is the dimer, a reminiscence of the high propensity for $\mathrm{Ar}_{n}$ clusters to form $\mathrm{Ar}_{2}^{+}$ions upon gas phase ionization. ${ }^{19,20}$ Some $\mathrm{Ar}_{4}^{+}$ions are detected but they come from secondary geminate recombinations ${ }^{22}$ of the dopant possibly favored by helium evaporation, as detailed later.

$\mathrm{Ar}_{2}^{+} \mathrm{He}_{q}$ and $\mathrm{Ar}_{3}^{+} \mathrm{He}_{q}$ are more abundant than the bare ions $\mathrm{Ar}_{2}^{+}$and $\mathrm{Ar}_{3}^{+} . \mathrm{Ar}_{4}^{+} \mathrm{He}_{q}$ are also present in mass spectra, especially at the early times of the dynamics, but the ionic dopant is a very loose $\mathrm{Ar}_{2}^{+} \cdots \mathrm{Ar} \cdots \mathrm{Ar}$ or $\mathrm{Ar}_{3}^{+} \cdots$ Ar complex and its abundance progressively vanishes during the 0.5 nanosecond of the simulations.

Based on abundances, we can divide up $\mathrm{Ar}_{n}^{+} \mathrm{He}_{q}$ fragments into three families: small fragments $(q \leq 50)$ which, as will be discussed in Section IIID, are mainly due to fast ejections of an ionic argon cluster, large fragments $(q>500)$ which will be shown in the same 
section to be signatures of trapped ionic argon clusters, and intermediate-sized fragments $(50<q \leq 500)$.

A histogram of the Ar-Ar distances, when the ionic dopant is trapped in a large helium cluster $(q>500)$ at the end of the 500-ps simulation, is depicted in Figure 5. When only two argon atoms remain trapped, the ionic core is $\mathrm{Ar}_{2}^{+}$with an average $\mathrm{Ar}$-Ar distance of $2.45 \AA$, very close to the equilibrium distance of the gas phase dimer in its ground electronic state $\left(R_{e q} \approx 2.40 \AA\right)$. However, when more argon atoms are trapped in what remains of the droplet, they do not necessarily form a bound argon cluster and the average Ar-Ar distance increases: $9.4 \AA$ for three argon atoms and $13.3 \AA$ for four argon atoms. A closer inspection of the histograms for $\mathrm{Ar}_{3}^{+}$or $\mathrm{Ar}_{4}^{+}$dopants reveals a peak around the equilibrium $\mathrm{Ar}-\mathrm{Ar}$ distance of $\mathrm{Ar}_{2}^{+}$at $2.40 \AA$, but no peak arises at twice this distance as could be expected for linear $\mathrm{Ar}_{3}^{+}$ionic cores (the most stable structure for $\mathrm{Ar}_{3}^{+}$in the gas phase). If any $\mathrm{Ar}_{3}^{+}$ionic core exists it is rather vibrationally excited and far from its linear equilibrium configuration. The width of the peak around $2.40 \AA$ can therefore be due to warmer $\mathrm{Ar}_{2}^{+}$or warm $\mathrm{Ar}_{3}^{+}$ core.

More specifically, in the case where the remaining ionic dopant is $\mathrm{Ar}_{3}^{+}$, the peak around $3 \AA$ roughly represents one third of the number of Ar-Ar distances. The other two thirds mainly correspond to much larger distances, with a distribution extending from 9 to $21 \AA$. Hence, the ionic dopant is rather a combination of $\mathrm{Ar}_{2}^{+}$and a roaming, nearly free, additional Ar atom. In the case where the remaining ionic dopant is $\mathrm{Ar}_{4}^{+}$, only $26 \%$ of the distances lie within the range of $\operatorname{Ar}_{n}^{+}$equilibrium distances $(2 \leq n \leq 4)$, namely $2-4 \AA$. The predominance of roaming argon atoms is thus also confirmed in this case, although discriminating between an $\mathrm{Ar}_{2}^{+}$or $\mathrm{Ar}_{3}^{+}$core is rather involved.

In order to validate our interpretation of the presence of argon atoms roaming inside the droplet, we first have to rule out the possibility that they are simply loose structures formed by the clustering of "snowballs" rigidly attached to the argon atoms, and in particular to the ones belonging to the ionic core, which would prevent the formation of compact argon clusters. Such structures were found in a recent ${ }^{4} \mathrm{He}-\mathrm{TDDFT}$ theoretical study ${ }^{78}$ on the dynamics of argon atoms clustering by collision with helium nanodroplets. Figure 6 displays the radial distributions of Ar-He distances between argon atoms, isolated or belonging to the ionic core of the cluster (i.e., $\mathrm{Ar}_{2}^{+}$or $\mathrm{Ar}_{3}^{+}$), and all helium atoms of the system at the end of the dynamics $(t=500 \mathrm{ps})$. The resulting distributions are similar to the ones obtained 
for $\mathrm{Cs}^{+}$or $\mathrm{Rb}^{+}$solvated in helium nanodroplets, ${ }^{79}$ with a small shell of helium atoms (the number of helium atoms is estimated at 12 about an $\mathrm{Ar}_{2}^{+}$core, 15.5 about an $\mathrm{Ar}_{3}^{+}$core, and 10 about an isolated argon atom) with radius $3.5-4 \AA$ about the ion and $4-4.5 \AA$ about the neutral argon atoms. These solid-like first shells about the dopant atoms could account for Ar-Ar distances of the order of 10-13 $\AA$ (taking into account the equilibrium He-He distance of $\sim 4.4 \AA$ ) found in Figure 5. However, the rest of the distribution at larger distances, up to $21 \AA$ for $\mathrm{Ar}_{3}^{+}$dopants and $23 \AA$ for $\mathrm{Ar}_{4}^{+}$dopants, is clearly due to the roaming of argon atoms. Hence, the resulting picture is that of an ionic core surrounded by a rigid snowball, and one or two neutral argon atoms (possibly also surrounded by a snowball) roaming inside the droplet.

Visual inspection of the configurations subject to ion trapping at the end of the simulation revealed that the dopant is mainly $\mathrm{Ar}_{2}^{+}$(40\%) and $\mathrm{Ar}_{2}^{+}+\mathrm{Ar}$ (42.5\%), with smaller contributions of warm (triangular) $\mathrm{Ar}_{3}^{+}$(2.5\%), $\mathrm{Ar}_{2}^{+}+\mathrm{Ar}+\mathrm{Ar}(10 \%)$, warm $\mathrm{Ar}_{3}^{+}+\mathrm{Ar}$ (2.5\%) and $\mathrm{Ar}_{2}^{+}+\mathrm{Ar}_{2}$ (2.5\%). The affinity for charge localization on $\mathrm{Ar}_{2}^{+}$can be confirmed by noting that, after $\mathrm{Ar}_{4}^{+}$has decayed to its ground electronic state, $97 \%$ of the charge is carried by only two argon atoms, on average. ${ }^{19}$ The other argon atoms can (i) dissociate and get expelled from the droplet; (ii) dissociate but slow down because of the surrounding helium atoms and remain in the droplet; or (iii) get stabilized and join the $\mathrm{Ar}_{2}^{+}$core to build an $\mathrm{Ar}_{3}^{+}$cluster (no compact $\mathrm{Ar}_{4}^{+}$cluster has been observed). Note that mass spectrometry experiments cannot discriminate either between a compact $\mathrm{Ar}_{3}^{+}$or $\mathrm{Ar}_{4}^{+}$cluster and an $\mathrm{Ar}_{2}^{+}$core with one or two argon atoms roaming within the shrunk droplet. Hence, the large argon-doped droplets detected in the mass spectra of Figure 3 at $t=500$ ps contain $\mathrm{Ar}_{2}^{+}$and $(n-2)$ Ar atoms roaming in the droplet rather than a compact $\mathrm{Ar}_{n}^{+}$cluster. However, these roaming argon atoms may recombine with the $\mathrm{Ar}_{2}^{+}$core. The experimental timescale being much longer than our simulations, there is more time for the system to relax, recombine or get ejected, which should account for some deviations between theoretical and experimental mass spectra.

\section{Characteristic times of the dynamics}

In this section we discuss qualitatively the sequential fragmentation dynamics of $\mathrm{Ar}_{4}^{+} \mathrm{He}_{1000}$ by providing approximate characteristic times deduced from exponential fitting (Figure 7a) 
or linear fitting (Figures $7 \mathrm{~b}$ and $7 \mathrm{c}$ ) of the curves representing the time evolution of relative fragment abundances. A more quantitative kinetic analysis will be performed in future works, when optimization of the programs will make it feasible to increase the number of trajectories by a significant factor in order to get better statistics.

At the earlier times of the dynamics, the abundance of $\mathrm{Ar}_{4}^{+} \mathrm{He}_{q}(q>500)$ rapidly decreases with an approximate lifetime of 15 ps. The quick disappearance of these large $\mathrm{Ar}_{4}^{+} \mathrm{He}_{q}$ clusters is related to electronic relaxation of the $\mathrm{Ar}_{4}^{+}$dopant that may release $\sim 1.5 \mathrm{eV}$ within the droplet since the global minimum of $\mathrm{Ar}_{2}^{+}$, the main ionic argon core observed in mass spectra, is about $-1.39 \mathrm{eV}$ with respect to the dissociation into $\operatorname{Ar}^{+}\left({ }^{2} \mathrm{P}\right)+\operatorname{Ar}^{19}$ This electronic relaxation is often followed by the ejection of one or two neutral argon atoms that yields the production of large fragments containing $\mathrm{Ar}_{2}^{+} \mathrm{or}_{\mathrm{Ar}_{3}^{+}}$, as shown by the sharp increases of the abundances in large $\mathrm{Ar}_{n}^{+} \mathrm{He}_{q}(n=2-3, q>500)$ fragments in Figure $7 \mathrm{~b}$. The formation of these $\mathrm{Ar}_{n}^{+} \mathrm{He}_{q}$ fragments starts at about $t \lesssim 5 \mathrm{ps}$ and evolves with characteristic times of 86 ps for $\mathrm{Ar}_{2}^{+} \mathrm{He}_{q}$ and $19 \mathrm{ps}$ for $\mathrm{Ar}_{3}^{+} \mathrm{He}_{q}$. The corresponding ionic dopants, namely $\mathrm{Ar}_{2}^{+}$and $\mathrm{Ar}_{3}^{+}$, can in turn be ejected from the large $\mathrm{Ar}_{n}^{+} \mathrm{He}_{q}$ fragments as obvious from the fast productions of small $\mathrm{Ar}_{2}^{+} \mathrm{He}_{q}$ and $\mathrm{Ar}_{3}^{+} \mathrm{He}_{q}(0<q \leq 50)$ fragments in Figure 7c. The formation of these small fragments starts at $t \approx 10$ ps and grows with characteristic times of 82 ps and 107 ps, respectively.

A closer inspection of the decay of $\operatorname{Ar}_{n}^{+} \mathrm{He}_{q}(q>500)$ clusters points to the secondary dissociation of $\mathrm{Ar}_{3}^{+}$dopants into $\mathrm{Ar}_{2}^{+}$as origin of most of the small $\mathrm{Ar}_{n}^{+} \mathrm{He}_{q}$ fragments: the curve for $\mathrm{Ar}_{3}^{+} \mathrm{He}_{q}(q>500)$ clusters drops from $50 \%$ to $20 \%$ between $t \approx 10 \mathrm{ps}$ and $t \approx 40 \mathrm{ps}$ parallel to the increase of $\mathrm{Ar}_{2}^{+} \mathrm{He}_{q}$ and $\mathrm{Ar}_{3}^{+} \mathrm{He}_{q}(0<q \leq 50)$ abundances from $0 \%$ to $\sim 40 \%$ within the first $40 \mathrm{ps}$. However, some of the small fragments must also originate from very fast dissociation of $\mathrm{Ar}_{4}^{+}$in large $\mathrm{Ar}_{4}^{+} \mathrm{He}_{q}$ clusters, whether sequential or not, in order to explain these increases of $\operatorname{Ar}_{n}^{+} \mathrm{He}_{q}(n=2-3,0<q \leq 50)$ abundances.

After $t \gtrsim 40 \mathrm{ps}$, the abundance of large $\operatorname{Ar}_{n}^{+} \mathrm{He}_{q}(2 \leq n \leq 4)$ fragments only slightly decreases (characteristic decay times of about $11.7 \mathrm{~ns}$ and $4.3 \mathrm{~ns}$ for $\mathrm{Ar}_{2}^{+} \mathrm{He}_{q}$ and $\mathrm{Ar}_{3}^{+} \mathrm{He}_{q}$, respectively) both due to the rarefaction of fast ejections and the long-term trapping of ionic dopants. Helium atom evaporation therefore becomes the main fragmentation process governing the dynamics. In particular, bare $\mathrm{Ar}_{2}^{+}$and $\mathrm{Ar}_{3}^{+}$fragments seem to originate from gradual helium atom evaporation from $\mathrm{Ar}_{3}^{+} \mathrm{He}_{q}$ and $\mathrm{Ar}_{2}^{+} \mathrm{He}_{q}$, as their abundances start increasing distinctly slower at $t \gtrsim 20-30$ ps. The abundance in $\mathrm{Ar}_{2}^{+}$keeps increasing, as 
expected owing to the evaporation of helium atoms from small $\mathrm{Ar}_{2}^{+} \mathrm{He}_{q}$ fragments, while bare $\mathrm{Ar}_{3}^{+}$fragments reach a plateau somewhat parallel to that of small $\mathrm{Ar}_{3}^{+} \mathrm{He}_{q}$ fragments.

Finally, it is worth noting that a small abundance of $\mathrm{Ar}_{4}^{+} \mathrm{He}_{q}(q>500)$ persists at long times. As explained before, this is not a compact $\mathrm{Ar}_{4}^{+}$cluster but rather an $\mathrm{Ar}_{2}^{+}$or a warm $\mathrm{Ar}_{3}^{+}$ionic core with two or one argon atom(s) roaming within the droplet. Droplet evaporation then makes it more and more likely for the roaming neutral argon atoms to meet the ionic core and recombine, the energy released by this recombination inducing helium evaporation and possible additional argon-cluster fragmentation. The slow decrease of $\mathrm{Ar}_{4}^{+} \mathrm{He}_{q}$ abundance at later times is due to these processes.

\section{Fast ejections and long-term trappings}

Based on the elements already discussed (kinetics of fragment appearances, Ar-Ar distances for trapped ion cores), three types of dynamical processes emerge:

1. Fast ejection of a small ionic fragment. This process, already observed in the simulation of neon-doped helium clusters ionization, ${ }^{37,38}$ occurs within the first 100 ps. "Small ionic fragments" refers to $\mathrm{Ar}_{n}^{+} \mathrm{He}_{q}$ with $q \leq 50$.

2. Long-term ion trapping. An ionic dopant remains in helium droplets of at least 500 helium atoms for more than 500 ps. This longer-term dynamics can involve possible recombinations and/or dopant release in the gas phase, leading to ionic fragments with a broad distribution of helium atoms observed at the end of our 500 ps simulations. This longer timescale is reminiscent of the nanosecond time scale estimated experimentally for helium evaporation and $\mathrm{CH}_{3} \mathrm{I}$ recombination following $\mathrm{CH}_{3} \mathrm{IHe}_{N}(\mathrm{~N}=2000-20$ 000) photodissociation. ${ }^{31}$

3. Intermediate processes. They include all the other processes, namely fragmentation processes that release small ionic fragments in the gas phase after $100 \mathrm{ps}$ (eg, delayed ejections and recombinations) and ion trappings in small nanodroplets (containing a few hundreds helium atoms at $t=500 \mathrm{ps}$ ) that might lead to recombinations.

Fast ejections and long-term trappings are illustrated in Figure 8. The upper part shows a trajectory in which $\mathrm{Ar}_{2}^{+}$is ejected, with a small number of helium atoms attached. $\mathrm{Ar}_{4}^{+}$ 
starts fragmenting inside the droplet at $t \approx 6.3 \mathrm{ps}$ into $\mathrm{Ar}_{3}^{+}+\mathrm{Ar}$, then into $\mathrm{Ar}_{2}^{+}+2 \mathrm{Ar}$ $(t \approx 8 \mathrm{ps})$, and the recoil energy is high enough for $\mathrm{Ar}_{2}^{+}$to leave the droplet, which occurs at $t \approx 20$ ps. It is accompanied by several helium atoms but, since it is vibrationally excited, the helium atoms keep dissociating. Indeed, visual inspection of the trajectory frame by frame shows that the ejected ionic fragment is $\mathrm{Ar}_{2}^{+} \mathrm{He}_{2}$ at $t=50 \mathrm{ps}$ and $\mathrm{Ar}_{2}^{+}$at $t>100 \mathrm{ps}$.

The lower part of Figure 8 shows a trajectory in which $\mathrm{Ar}_{2}^{+}$remains trapped inside the droplet. As in the previous trajectory, $\mathrm{Ar}_{4}^{+}$dissociates very early on into $\mathrm{Ar}_{3}^{+}+\mathrm{Ar}(t \approx 2 \mathrm{ps})$ and then into $\mathrm{Ar}_{2}^{+}+2 \mathrm{Ar}(t \approx 7 \mathrm{ps})$. However, in this case, $\mathrm{Ar}_{2}^{+}$is no longer ejected; most of the recoil energy is taken away by the two neutral argon fragments which leave the droplet, while $\mathrm{Ar}_{2}^{+}$remains trapped inside (final snapshot at $t \approx 13 \mathrm{ps}$ ). $\mathrm{Ar}_{2}^{+}$internal excitation is then slowly cooled down through helium atom evaporation, leading to a gradual decrease of the droplet size.

The difference between these two processes is also clearly visible from the kinetic energy of the $\mathrm{Ar}_{2}^{+}$center of mass: $643 \mathrm{~cm}^{-1}(926 \mathrm{~K})$ for the former and $21 \mathrm{~cm}^{-1}(30 \mathrm{~K})$ for the latter.

Figure 9 shows an example of intermediate process involving several fragmentations and recombinations. $\mathrm{Ar}_{4}^{+}$fragmentation occurs somewhat later in this case since it takes $\sim 30 \mathrm{ps}$ to get $\mathrm{Ar}_{2}^{+}+2 \mathrm{Ar}$. Then, the argon atoms and the $\mathrm{Ar}_{2}^{+}$cluster remain inside the droplet which starts shrinking by evaporating helium atoms. The argon atoms are thus roaming inside a smaller and smaller droplet and a first recombination, $\mathrm{Ar}_{2}^{+}+\mathrm{Ar} \rightarrow \mathrm{Ar}_{3}^{+}$, occurs at $t \approx 220$ ps. However, the newly formed $\mathrm{Ar}_{3}^{+}$ion is too warm and cannot be cooled fast enough by the surrounding helium atoms, so it dissociates again into $\mathrm{Ar}_{2}^{+}+$Ar at $t \approx 225 \mathrm{ps}$. The helium droplet keeps shrinking even more and one of the two neutral argon atoms leaves the droplet, the other remains trapped and eventually recombines with the ionic $\mathrm{Ar}_{2}^{+}$core to form $\mathrm{Ar}_{3}^{+}$at $t \approx 395 \mathrm{ps}$. The internal energy of $\mathrm{Ar}_{3}^{+}$is dissipated inside what remains of the droplet and a linear $\mathrm{Ar}_{3}^{+}$cluster is finally released in the gas phase at $t \approx 420 \mathrm{ps}$.

In order to quantify the importance of the three processes identified above, we have classified the trajectories with respect to their propensity for fast ejection, long-term ion trapping or intermediate processes. This sorting is of course somewhat arbitrary, but the two extreme cases are clear and intuitive. Quantitatively, 56.3\% of the trajectories lead to fast ejections, $25.3 \%$ to long-term trappings, and $18.4 \%$ correspond to intermediate processes, these percentages being subject to variations depending on the effective He-He potential or velocity 
adjustment technique considered (see Figure S6 of the Supplementary Material). These percentages can be simply derived from Figure 10a by summing over all the percentages of trajectories for a given dynamical process (fast ejection, long-term trapping, or intermediate process). As can be seen in that figure, fast ejection is on average the most probable event in these simulations. It is about twice as probable as long-term trapping when summed over all electronic states. It is also clear from Figure 10a that the highest initial energy states are more prone to fast ejection, like the trajectory in the top plot of Figure 8 which was initiated on the highest electronic state. On the other hand, the lowest-lying electronic states tend to favor trapping like the trajectory in the bottom plot of Figure 8 which was initiated on the lowest electronic state. Intermediate processes are distributed somewhat uniformly over the whole energy range, with a local maximum for state 5 . This overall picture could somewhat be expected since, at time $t=0, \mathrm{Ar}_{4}^{+}$has by definition more available energy in the highest electronic states than in the lowest ones. However, it is worth noting that the probability for ejection is not negligible for the lowest initial electronic states. In this case, visual examination of the corresponding trajectories revealed that if $\mathrm{Ar}_{2}^{+}$is the ejected ion, it always brings along several helium atoms, and the droplet left behind is relatively intact. Some neutral argon atoms can even remain trapped in the droplet, generally as single argon atoms although dimers were also found. $\mathrm{Ar}_{3}^{+}$(linear or not) can be ejected as a bare ion even when the initial electronic state is one of the lowest ones, but it can also bring along helium atoms.

Trapping covers a broad range of processes that account for the width of the distribution of peaks observed in the theoretical mass spectra of Figure 3. When the $\mathrm{Ar}_{4}$ dopant is ionized to its lower energy states, its internal energy may not be sufficient to eject all the neutral argon atoms upon fragmentation. The ionic core (usually $\mathrm{Ar}_{2}^{+}$) may remain trapped within the nanodroplet together with one or two argon atoms, and is then subject to later recombinations as the droplet shrinks due to helium atoms evaporation. This is what happened in the trajectory presented in Figure 9. Ionization to the upper energy states can also lead to ion trapping, but the helium droplet severely shrinks as many helium atoms evaporate to dissipate the internal energy. The droplet size is then below 500 and the trajectory is classified among intermediate processes.

The ionic core of the droplet can recombine with argon atoms if they were not ejected. This recombination process releases a lot of energy in the droplet, which induces even more 
helium evaporation. This can eventually result in the gas phase release of $\mathrm{Ar}_{3}^{+}$or $\mathrm{Ar}_{4}^{+}$, possibly surrounded by a few helium atoms. This influence of droplet shrinking, limiting the volume in which fragments can move and thereby inducing dynamics pathways different from the gas phase, is known for instance in proteomics where doped droplets are ionized by

electrospray ionization. In this case, as proposed by the charged residue model ${ }^{80,81}$ solvent evaporation induces higher charge density which in turn leads to further fragmentation.

To provide a more precise picture of the relationship between the initial excitation of the dopant and the mechanisms of ion formation, Figure 10b reports the mass of the ionic fragments at $t=500 \mathrm{ps}$ as a function of the initial $\mathrm{Ar}_{4}^{+}$electronic state populated. As previously discussed fast ejections (red symbols) can occur for a wide range of initial energies, although the higher ones are preferred. Two large fragments, whose masses do not lie within the range allowed by the definition of fast ejections (ie, less than 50 helium atoms), are also attributed to this fragmentation mechanism (see electronic states 10 and 11) which can appear contradictory. They correspond to small ions ejected after an extensive droplet fragmentation before $t=100 \mathrm{ps}$ but captured later by one helium cluster in its course to get a more stable spherical shape. This is an example of the wide variety of processes at play that can hardly be interpreted from a single analysis of mass spectra, the final ionic fragment mass being not always representative of the fragmentation process primarily triggered upon ionization of the doped helium nanodroplet. Figure 10b also reveals that the trapping of $\mathrm{Ar}_{2}^{+}$is favored by the higher electronic states, whereas trapped $\mathrm{Ar}_{3}^{+}$and $\mathrm{Ar}_{4}^{+}$(or lightly bound complexes like $\mathrm{Ar}_{2}^{+} \cdots \mathrm{Ar}$ and $\mathrm{Ar}_{2}^{+} \cdots \mathrm{Ar} \cdots \mathrm{Ar}$ ) are more common at lower energies. Intermediate processes yield fragment masses in between fast ejections and long-term trappings and $\mathrm{Ar}_{n}^{+}$-containing ions $(n=3-4)$ remain favored by the lower-energy states.

These observations enable us to predict that for larger $\operatorname{Ar}_{n}^{+}$dopants $(n>4)$, cycles of recombinations and helium evaporations will yield mass spectra significantly different from those observed in the gas phase.

\section{E. Kinetic energy of fragments}

In a previous work on neon clusters, ${ }^{38}$ the analysis of helium kinetic energy distributions revealed a biexponential behavior that was attributed to the existence of two different frag- 
mentation processes to account for the loss of helium atoms, namely thermal evaporation and ejection. Thermal evaporation was characterized by a kinetic energy release of 3-7 $\mathrm{cm}^{-1}$ /atom, while helium atom ejection corresponded to much faster helium atoms with kinetic energies of $28-45 \mathrm{~cm}^{-1}$. The latter was attributed to fast ejection of the embedded ionic neon fragment, leading to energetic collisions with helium atoms, while the former was attributed to common helium atom evaporation that typically removes $5 \mathrm{~cm}^{-1}$ from the droplet for each helium atom lost. The ability for helium atoms to dissipate more than their binding energy to the droplet upon ejection was already known from experimental work on $\mathrm{TPM}^{27}$ and $\mathrm{HCN},{ }^{28}$ where the first thousands helium atoms were found to remove on average $22 \mathrm{~cm}^{-1} /$ atom and $19 \mathrm{~cm}^{-1} /$ atom, respectively. They correspond to "prompt" atom emission observed in ${ }^{4} \mathrm{He}-\mathrm{TDDFT}$ simulations. ${ }^{48}$

The helium kinetic energy distribution obtained in this work for $\mathrm{Ar}_{4}$ ionization inside a ${ }^{4} \mathrm{He}_{1000}$ droplet is plotted in Figure 11. There is a similarity with the case of neon cluster ionization inside a small (100 ${ }^{4} \mathrm{He}$ atoms) droplet, in that the low energy part of the distribution can be approximately fitted with a Boltzmann distribution corresponding to $4 \mathrm{~cm}^{-1} /$ atom, and there is a long tail extending to higher kinetic energies. However the whole distribution could not be fitted by a simple biexponential function. We believe that this is because evaporation and ejection are not the only fragmentation processes at play in larger droplets. When the ionic dopant is trapped, there can be different processes: fragmentation during and after electronic relaxation, helium atom evaporation to cool down the droplet and the dopant, possible recombinations after some roaming period of neutral argon atoms, which can in turn lead to new fragmentations. Hence, it is not very surprising that the kinetic energy distribution of the dissociating helium atoms could not be fitted to a simple analytical expression. However, we can assert that helium atoms evaporated during fast ejections are, on average, a bit more energetic $\left(\sim 8-9 \mathrm{~cm}^{-1} /\right.$ atom $)$ than those evaporated during long-term trapping $\left(\sim 6 \mathrm{~cm}^{-1}\right)$. Long-term trapping is characterized by thermal evaporation of helium atoms once the energy due to electronic relaxation has been dissipated. On the contrary, ejections can severely destabilize the droplet, by creating a partial burst of the droplet, and the energy of departing helium atoms is a reflect of the remaining large helium clusters rearrangements (as spheres to minimize their surface tension) and of the later progressive helium evaporation.

Since helium kinetic energy distributions could not unambiguously discriminate between 
thermal evaporation, ion ejection, and trapping, we turned to an alternate observable, the translational energy of fragments. This is what Braun et al. used to investigate the photodissociation dynamics of alkyl iodides in helium nanodroplets. ${ }^{29,30}$ For each group of trajectories (i.e., those subject to fast ejection, long-term trapping, or intermediate processes) we calculated the average center-of-mass kinetic energy of the ejected or embedded charged argon cluster. Focusing on the center of mass enables us to discard the vibrational energy of argon complexes, such as $\mathrm{Ar}_{2}^{+} \cdots \mathrm{Ar} \cdots \mathrm{Ar}$, trapped in helium nanodroplets. The average translational energy of ejected ions is $72 \mathrm{~cm}^{-1}$ in our simulations, whereas that of the trapped species is only $\sim 1.3 \mathrm{~cm}^{-1}$. Intermediate processes correspond to a translational energy of $\sim 7-9 \mathrm{~cm}^{-1}$, which is in between. Hence, the translational energy of the ions can be used as a criterion to distinguish between ejection and trapping: it gives a clearer signature than the velocity of evaporating helium atoms.

\section{DISCUSSION AND CONCLUSION}

In this work we have studied the fragmentation dynamics following electron-impact ionization of $\mathrm{Ar}_{4}$ inside a $1000-{ }^{4} \mathrm{He}$ atom droplet, using a mixed quantum-classical simulation in order to describe electronic transitions in $\mathrm{Ar}_{4}^{+}$, and the ZPAD method to take into account, at least approximately, quantum delocalization effects for helium atoms. Our simulations have revealed that the parent ions electronically relax on a picosecond time scale before fragmenting, as expected from their gas phase behavior. $\operatorname{Ar}_{n}^{+}(n=2$ or 3 ) can be ejected from the droplet, as observed in the case of neon cluster ionization in smaller $100-{ }^{4} \mathrm{He}$ atom droplets, ${ }^{37,38}$ or be cooled through helium atom evaporation. They have also evidenced three dynamical processes: fast ejection of small ionic fragments, long-term ion trapping, and intermediate processes.

The most original result is the occurrence of a rich variety of trapping processes, in which an $\mathrm{Ar}_{n}^{+}$ion (mostly $\mathrm{Ar}_{2}^{+}$) can be trapped together with one or two neutral roaming argon atoms in a shrinking helium droplet: this possibly yields recombinations and further dissociations, which in turn can release a small argon ion in the gas phase. To the best of our knowledge, this is the first theoretical work evidencing trapping and recombination. The fact that these processes were not observed in previous studies on chlorine photodissociation or dissociative ionization of small neon clusters in helium droplets is due to the fact that the 
droplet size was limited to $\sim 100-200$ helium atoms in order to include realistic quantum effects in the simulations, ${ }^{32,37,38}$ or that the kinetic energy of the departing atoms was too high. ${ }^{33}$ The importance of helium atom cooling and confinement inside a shrinking droplet can only be reproduced for larger helium nanodroplets like those considered in the present work.

Extending our studies to larger dopant sizes would also provide valuable clues to correlate the fragments observed in experimental mass spectra with parent cluster sizes. In addition, typical helium nanodroplet sizes range between $10^{3}$ and $10^{6}$ helium atoms, or even up to $10^{11}$ in recent applications devoted to quantum vortex lattices ${ }^{82}$ or silver doping. ${ }^{83}$ Improving our simulation method for handling much larger droplets, for instance by implementing "smart" cutoff distances to neglect long-range He-He interactions, will therefore become a requirement in the near future. In our mixed quantum-classical approach, a surface hopping method is coupled to a DIM model, corrected by adding induced dipole-induced dipole interactions, and effective He-He interactions modified to take into account average zero-point effects. No significant effect of the velocity adjustment techniques has been identified but changes in atom-atom interaction potentials do have an influence on the relative propensity for ejections and trappings (see Figures S2-S7 of the Supplementary Material). More sophisticated methods that would include spin-orbit couplings in the DIM matrix, ${ }^{41,84-86}$ or quantum effects in the He-Ar and $\mathrm{He}_{-} \mathrm{Ar}^{+}$interaction potentials, might be needed to get a more precise picture of the fragmentation dynamics of argon-doped nanodroplets.

This work constitutes the first step toward a realistic modeling of the dynamics of large doped helium nanodroplets upon electron impact ionization. The wealth of observed processes, from small fragment ejection to trapping, geminate recombination and helium evaporation extending on the nanosecond time scale, make $\mathrm{Ar}_{4}^{+} \mathrm{He}_{1000}$ a valuable prototype system to evaluate the relevance of our dynamical approach. However, experiments deal with a wide size distribution of argon clusters and helium nanodroplets. In particular, the increased intensity at the mass of $\mathrm{Ar}^{+} \mathrm{He}_{12}, \mathrm{Ar}^{+} \mathrm{He}_{32}$, and $\mathrm{Ar}^{+} \mathrm{He}_{44}$ observed in experimental data, which was attributed to an increased stability of the icosahedral structure of helium atoms about an $\mathrm{Ar}^{+}$ion, ${ }^{44}$ would require additional simulations on smaller dopant sizes (i.e., $\mathrm{Ar}_{n}^{+}$, $n \leq 3)$ to check whether such fragments only originate from the ionization of embedded argon monomers or can be produced by ionizing $\mathrm{Ar}_{2}$ or $\mathrm{Ar}_{3}$.

Large neutral $\mathrm{ArHe}_{q}$ and $\mathrm{Ar}_{2} \mathrm{He}_{q}$ fragments were also observed in our 0.5 ns simulations, 
although in much lower abundance than helium atoms (see Figure S3 of the Supplementary Material). Evaluating the probability for such clusters to be ionized in experimental setups might provide additional clues to rationalize the large amount of $\mathrm{Ar}^{+} \mathrm{He}_{q}$ fragments detected in mass spectrometers.

Although complete electronic relaxation of $\mathrm{Ar}_{4}^{+}$was achieved within a few tens of picoseconds and most of ion ejections occurred within one hundred picoseconds, the dynamics of $\mathrm{Ar}_{4}^{+} \mathrm{He}_{1000}$ clusters may last nanoseconds or more since the systems were not fully thermalized after 500 ps simulations. This is in contrast with small neon-doped helium nanodroplets whose fragmentation dynamics was generally completed within $\sim 30$ ps owing to the ejection of ions. ${ }^{38}$ This longer timescale comes from ion trapping and can be confirmed by following the time evolution of fragment abundances. Trapped species include $\mathrm{Ar}_{2}^{+}, \mathrm{Ar}_{3}^{+}$, and $\mathrm{Ar}_{4}^{+}$ but the latter two are slightly bound $\mathrm{Ar}_{2}^{+} \cdots \operatorname{Ar}$ and $\mathrm{Ar}_{2}^{+} \cdots \operatorname{Ar} \cdots \operatorname{Ar}$ complexes, or an $\mathrm{Ar}_{2}^{+}$ ion with one or two neutral argon atoms roaming around it, rather than the corresponding compact clusters. The helium nanodroplet then acts as a confined low-temperature medium that can favor the recombination of an ionic complex that would otherwise dissociate in the gas phase. Moreover, a number of experimental studies have stressed the nonthermal cooling exerted by the first thousands evaporating helium atoms. ${ }^{27-30}$ In the simulations presented here, there is a significant proportion of helium atoms which dissociate with a kinetic energy higher than expected from thermal evaporation. This occurs upon ion ejection or early dopant electronic relaxation. The average energy taken away by each helium atom can be up to $10 \mathrm{~cm}^{-1}$.

In addition, we have found that the kinetic energy of the center of mass of the $\mathrm{Ar}_{n}^{+} \mathrm{He}_{q}$ fragments could be used as a characteristics of the dynamical process: fast ejection of small ionic fragments, long-term ion trapping, or intermediate processes. For all these reasons, comparison with an experiment using velocity map imaging of the fragments would be very valuable.

\section{SUPPLEMENTARY MATERIAL}

The effective He-He interaction potential obtained for a wavefunction cutoff radius $R=$ $1.5 \AA$ is plotted in Figure S1 and the corresponding parameters fitted on Eq.(3) are collected in Table S1. The influence of the He-He interaction potential and velocity adjustment tech- 
nique is discussed on the basis of polar plots for abundances of ionic and neutral fragments at $t=500$ ps (Figures S2 and S3), relaxation times (Figure S4), time evolution of $\mathrm{Ar}_{n}^{+} \mathrm{He}_{q}$ relative abundances (Figure S5), and figures showing the propensity for the different dynamical processes as a function of the initial $\mathrm{Ar}_{4}^{+}$electronic state number (Figures S6 and S7).

\section{DATA AVAILABILITY STATEMENT}

The data that support the findings of this study are available from the corresponding author upon reasonable request.

\section{ACKNOWLEDGMENTS}

Prof. Marius Lewerenz is gratefully acknowledged for providing us with the set of routines devoted to the convergence of the effective He-He potential. DAB warmly acknowledges Dr Raphaël Vallon for logistical support and the regional supercomputing centre of ChampagneArdenne (ROMEO) for computational resources and technical support.

\section{Appendix: Derivative of atomic charges $q_{i}^{(j)}$}

Assuming an $\mathrm{Ar}_{4}^{+}$cluster excited in its adiabatic state $V_{j}$, the charge $q_{i}^{(j)}$ carried by argon atom $i$ is given by

$$
q_{i}^{(j)}=\sum_{\alpha \in\{x, y, z\}}\left|\left\langle p_{i \alpha} \mid \Phi_{j}\right\rangle\right|^{2}
$$

where $\left\{\left|p_{i \alpha}\right\rangle\right\}(i \in[1, n], \alpha \in\{x, y, z\})$ is the basis set of reference $p$ orbitals in the Cartesian space-fixed frame and $\left|\Phi_{j}\right\rangle$ is the $\operatorname{Ar}_{4}^{+}$eigenstate $j$. Since $\left\langle\frac{d p_{i \alpha}}{d x_{l}} \mid \Phi_{j}\right\rangle=0$

$$
\frac{d q_{i}^{(j)}}{d x_{l}}=2 \sum_{\alpha \in\{x, y, z\}}\left\langle p_{i \alpha} \mid \Phi_{j}\right\rangle\left\langle p_{i \alpha} \mid \frac{d \Phi_{j}}{d x_{l}}\right\rangle .
$$

Introducing the closure relationship,

$$
\begin{aligned}
\left\langle p_{i \alpha} \mid \frac{d \Phi_{j}}{d x_{l}}\right\rangle & =\left\langle p_{i \alpha}\left|\left(\sum_{k=1}^{3 n}\left|\Phi_{k}\right\rangle\left\langle\Phi_{k}\right|\right)\right| \frac{d \Phi_{j}}{d x_{l}}\right\rangle \\
& =\sum_{k=1}^{3 n}\left\langle p_{i \alpha} \mid \Phi_{k}\right\rangle\left\langle\Phi_{k}\left|\frac{d}{d x_{l}}\right| \Phi_{\mathrm{j}}\right\rangle=\sum_{k=1}^{3 n}\left\langle p_{i \alpha} \mid \Phi_{k}\right\rangle d_{k j}^{(l)}
\end{aligned}
$$


where $d_{k j}^{(l)}$ is the $l^{t h}$ component of the $3 n \times 3 n$ nonadiabatic coupling vector $\mathbf{d}_{k j}$. Finally, we conclude that

$$
\frac{d q_{i}^{(j)}}{d x_{l}}=2 \sum_{\alpha \in\{x, y, z\}} \sum_{k=1}^{3 n}\left\langle p_{i \alpha} \mid \Phi_{j}\right\rangle\left\langle p_{i \alpha} \mid \Phi_{k}\right\rangle d_{k j}^{(l)} .
$$

This expression is not fully analytical since the eigenstates $\left|\Phi_{j}\right\rangle$ are determined numerically by diagonalizing the DIM matrix of $\mathrm{Ar}_{4}^{+}$. However, its accuracy is confirmed by the proper energy conservation during the dynamics of $\mathrm{Ar}_{4}^{+} \mathrm{He}_{1000}$.

\section{REFERENCES}

${ }^{1}$ M. Hartmann, R. E. Miller, J. P. Toennies, and A. Vilesov, Phys. Rev. Lett. 75, 1566 (1995).

${ }^{2}$ J. Harms, M. Hartmann, B. Sartakov, J. P. Toennies, and A. F. Vilesov, J. Mol. Spectrosc. 185, 204 (1997).

${ }^{3}$ S. Grebenev, J. P. Toennies, and A. F. Vilesov, Science 279, 2083 (1998).

${ }^{4}$ K. Nauta and R. E. Miller, Science 283, 1895 (1999).

${ }^{5}$ G. Haberfehler, P. Thaler, D. Knez, A. Volk, F. Hofer, W. E. Ernst, and G. Kothleitner, Nat. Comm. 6, 8779 (2015).

${ }^{6}$ M. Lewerenz, B. Schilling, and J. P. Toennies, J. Chem. Phys. 102, 8191 (1995).

${ }^{7}$ R. Fröchtenicht, U. Henne, J. P. Toennies, A. Ding, M. Fieber-Erdmann, and T. Drewello, J. Chem. Phys. 104, 2548 (1996).

${ }^{8}$ B. E. Callicoatt, K. Förde, L. F. Jung, T. Ruchti, and K. C. Janda, J. Chem. Phys. 109, 10195 (1998).

${ }^{9}$ B. E. Callicoatt, K. Förde, T. Ruchti, L. Jung, K. C. Janda, and N. Halberstadt, J. Chem. Phys. 108, 9371 (1998).

${ }^{10}$ N. Halberstadt and K. C. Janda, Chemical Physics Letters 282, 409 (1998), see also erratum, 2016.

${ }^{11}$ J. H. Kim, D. S. Peterka, C. C. Wang, and D. M. Neumark, J. Chem. Phys. 124, 214301 (2006).

${ }^{12}$ H. Haberland, Surf. Sci. 156, 305 (1985).

${ }^{13}$ U. Buck, in The Chemical Physics of Atomic and Molecular Clusters, edited by G. Scoles (North Holland, Amsterdam, 1990) p. 543. 
${ }^{14}$ T. D. Märk and O. Echt, in Clusters of atoms and molecules, edited by H. Haberland (Springer, Berlin, 1994) pp. 154-182.

${ }^{15}$ A. Bastida, N. Halberstadt, J. A. Beswick, F. X. Gadéa, U. Buck, R. Galonska, and C. Lauenstein, Chem. Phys. Lett. 249, 1 (1996).

${ }^{16}$ P. Lohbrandt, R. Galonska, H. Kim, M. Schmidt, C. Lauenstein, and U. Buck, in Atomic and Molecular Beams: The State of the Art 2000, edited by R. Campargue (Springer, Berlin, 2000) pp. 623-636.

${ }^{17}$ D. Bonhommeau, A. Viel, and N. Halberstadt, J. Chem. Phys. 123, 054316 (2005).

${ }^{18}$ D. Bonhommeau, N. Halberstadt, and A. Viel, J. Chem. Phys. 124, 024328 (2006).

${ }^{19}$ D. Bonhommeau, N. Halberstadt, and A. Viel, J. Chem. Phys. 124, 184314 (2006).

${ }^{20}$ D. Bonhommeau, N. Halberstadt, and U. Buck, Int. Rev. Phys. Chem. 26, 353 (2007).

${ }^{21}$ R. J. Donnelly, Phys. Lett. 39A, 221 (1972).

${ }^{22}$ IUPAC distinguishes between primary geminate recombination, where recombination occurs before any separation by diffusion, and secondary geminate recombination where it occurs after the fragments have been separated and then come together by diffusion. The caging referred to in this work would be secondary geminate recombination, although some primary geminate recombination can also occur at very early times. IUPAC. Compendium of Chemical Terminology, 2nd ed. (the "Gold Book"). Compiled by A. D. McNaught and A. Wilkinson. Blackwell Scientific Publications, Oxford (1997). Online version (2019-) created by S. J. Chalk. ISBN 0-9678550-9-8. https://doi.org/10.1351/goldbook.

${ }^{23}$ A. Scheidemann, B. Schilling, and J. P. Toennies, J. Phys. Chem. 97, 2128 (1993).

${ }^{24}$ D. Stolyarov, E. Polyakova, and C. Wittig, J. Phys. Chem. A 108, 9841 (2004).

${ }^{25}$ D. M. Brink and S. Stringari, Z. Phys. D 15, 257 (1990).

${ }^{26}$ M. Iino, M. Suzuki, and A. J. Ikushima, J. Low Temp. Phys. 61, 155 (1985).

${ }^{27}$ W. K. Lewis, B. E. Applegate, J. Sztáray, B. Sztáray, T. Baer, R. J. Bemish, and R. E. Miller, J. Am. Chem. Soc. 126, 11283 (2004).

${ }^{28}$ W. K. Lewis, R. J. Bemish, and R. E. Miller, J. Chem. Phys. 123, 141103 (2005).

${ }^{29}$ A. Braun and M. Drabbels, Phys. Rev. Lett. 93, 253401 (2004).

${ }^{30}$ A. Braun and M. Drabbels, J. Chem. Phys. 127, 114303 (2007).

${ }^{31}$ A. Braun and M. Drabbels, J. Chem. Phys. 127, 114305 (2007).

${ }^{32}$ T. Takayanagi and M. Shiga, Chem. Phys. Lett 372, 90 (2003).

${ }^{33}$ A. Vilà, M. González, and R. Mayol, J. Chem. Theory Comput. 11, 899 (2015). 
${ }^{34}$ A. Vilà, M. González, and R. Mayol, Phys. Chem. Chem. Phys. 18, 2409 (2016).

${ }^{35}$ A. Vilà, M. González, and R. Mayol, Phys. Chem. Chem. Phys. 17, 32241 (2015).

${ }^{36}$ We call the dynamics "mixed quantum-classical" rather than "classical" since the latter usually refers to a specific method based on the JWKB approximation of the wave function, resulting from a development in powers of Planck's constant.

${ }^{37}$ D. Bonhommeau, P. T. Lake, Jr., C. L. Quiniou, M. Lewerenz, and N. Halberstadt, J. Chem. Phys. 126, 051104 (2007).

${ }^{38}$ D. Bonhommeau, M. Lewerenz, and N. Halberstadt, J. Chem. Phys. 128, 054302 (2008).

${ }^{39}$ A. Scheidemann, J. P. Toennies, and J. A. Northby, Phys. Rev. Lett. 64, 1899 (1990).

${ }^{40}$ T. Ruchti, K. Förde, B. E. Callicoatt, H. Ludwigs, and K. C. Janda, J. Chem. Phys. 109, 10679 (1998).

${ }^{41}$ C. A. Brindle, M. R. Prado, K. C. Janda, N. Halberstadt, and M. Lewerenz, J. Chem. Phys. 123, 064312 (2005).

${ }^{42}$ P. Bartl, S. Denifl, P. Scheier, and O. Echt, Phys. Chem. Chem. Phys. 15, 16599 (2013).

${ }^{43}$ T. K. Ha, P. Rupper, A. Wüest, and F. Merkt, Mol. Phys. 101, 827 (2003).

${ }^{44}$ P. Bartl, C. Leidlmair, S. Denifl, P. Scheier, and O. Echt, J. Phys. Chem. A 118, 8050 (2014).

${ }^{45}$ U. Buck and H. Meyer, Phys. Rev. Lett. 52, 109 (1984).

${ }^{46}$ U. Buck and H. Meyer, J. Chem. Phys. 84, 4854 (1986).

${ }^{47}$ M. Barranco, R. Guardiola, S. Hernandez, R. Mayol, J. Navarro, and M. Pi, J. Low Temp. Phys. 142, 1 (2006).

${ }^{48}$ F. Ancilotto, M. Barranco, F. Coppens, J. Eloranta, N. Halberstadt, A. Hernando, D. Mateo, and M. Pi, Int. Rev. Phys. Chem. 36, 621 (2017).

${ }^{49}$ T. Takayanagi and M. Shiga, Phys. Chem. Chem. Phys. 6, 3241 (2004).

${ }^{50}$ A. Wada, T. Takayanagi, and M. Shiga, J. Chem. Phys. 119, 5478 (2003).

${ }^{51}$ F. Calvo, Phys. Rev. B 95, 035429 (2017).

${ }^{52}$ E. J. Heller, J. Chem. Phys. 75, 2923 (1981), https://doi.org/10.1063/1.442382.

${ }^{53}$ M. Sterling, Z. Li, and V. A. Apkarian, J. Chem. Phys. 103, 5679 (1995).

${ }^{54}$ P. Slavíček, P. Jungwirth, M. Lewerenz, N. H. Nahler, M. Fárník, and U. Buck, J. Phys. Chem. A 107, 7743 (2003).

${ }^{55}$ The "temperature" is a kinetic temperature since the dynamics is microcanonical. The trajectory is periodically interrupted and velocities are rescaled if needed in order to maintain 
the temperature.

${ }^{56}$ R. A. Aziz, J. Chem. Phys. 99, 4518 (1993).

${ }^{57}$ M. Keil, L. J. Danielson, and P. J. Dunlop, J. Chem. Phys. 94, 296 (1991).

${ }^{58}$ C. Douketis, G. Scoles, S. Marchetti, M. Zen, and A. J. Thakkar, J. Chem. Phys. 76, 3057 (1982).

${ }^{59}$ A. R. Janzen and R. A. Aziz, J. Chem. Phys. 107, 914 (1997).

${ }^{60}$ O. Echt, D. Kreisle, E. Recknagel, J. J. Saenz, R. Casero, and J. M. Soler, Phys. Rev. A 38, 3236 (1988).

${ }^{61}$ C. L. Briant and J. J. Burton, J. Chem. Phys. 63, 2045 (1975).

${ }^{62}$ An error has been noticed in Ref 23 page 5 where $\tanh [a(R+b)]$ is indicated instead of $\tanh [a(R-b)]$ for the switching function $T(R)$.

${ }^{63}$ J. C. Tully, J. Chem. Phys. 93, 1061 (1990).

${ }^{64}$ S. Hammes-Schiffer and J. C. Tully, J. Chem. Phys. 101, 4657 (1994).

${ }^{65}$ F. O. Ellison, J. Am. Chem. Soc. 85, 3540 (1963).

${ }^{66}$ W. R. Wadt, Appl. Phys. Lett. 38, 1030 (1981).

${ }^{67}$ P. J. Kuntz and J. Valldorf, Z. Phys. D 8, 195 (1988).

68 "Argon electric dipole polarizability extracted from the NIST database on 19/03/2018 at url: https://cccbdb.nist.gov/exp2x.asp,".

${ }^{69}$ A. Carrington, C. A. Leach, A. J. Marr, A. M. Shaw, M. R. Viant, J. M. Hutson, and M. M. Law, J. Chem. Phys. 102, 2379 (1995).

${ }^{70}$ D. Bonhommeau, A. Viel, and N. Halberstadt, J. Chem. Phys. 120, 11359 (2004).

${ }^{71}$ N. C. Blais and D. G. Truhlar, J. Chem. Phys. 79, 1334 (1983).

${ }^{72}$ N. C. Blais, D. G. Truhlar, and C. A. Mead, J. Chem. Phys. 89, 6204 (1988).

${ }^{73}$ M. D. Hack, A. W. Jasper, Y. L. Volobuev, D. W. Schwenke, and D. G. Truhlar, J. Phys. Chem. A 103, 6309 (1999).

${ }^{74}$ R. W. Hamming, J. ACM 6, 37 (1959).

${ }^{75}$ D. A. Bonhommeau, J. Chem. Phys. 146, 124314 (2017).

${ }^{76}$ F. Laimer, L. Kranabetter, L. Tiefenthaler, S. Albertini, F. Zappa, A. M. Ellis, M. Gatchell, and P. Scheier, Phys. Rev. Lett. 123, 165301 (2019).

${ }^{77}$ I. Janeček, T. Janča, P. Naar, R. Kalus, and F. X. Gadea, J. Chem. Phys. 138, 044303 (2013). 
${ }^{78}$ F. Coppens, F. Ancilotto, M. Barranco, N. Halberstadt, and M. Pi, Phys. Chem. Chem. Phys. 21, 17423 (2019).

${ }^{79}$ A. Leal, D. Mateo, A. Hernando, M. Pi, M. Barranco, A. Ponti, F. Cargnoni, and M. Drabbels, Phys. Rev. B 90, 224518 (2014).

${ }^{80}$ J. B. Fenn, Angew. Chem. Int. Ed. 42, 3871 (2003).

${ }^{81}$ M. Dole, L. L. Mack, R. L. Hines, R. C. Mobley, L. D. Ferguson, and M. B. Alice, J. Chem. Phys. 49, 2240 (1968).

${ }^{82}$ L. F. Gomez, K. R. Ferguson, J. P. Cryan, C. Bacellar, R. M. P. Tanyag, C. Jones, S. Schorb, D. Anielski, A. Belkacem, C. Bernando, R. Boll, J. Bozek, S. Carron, G. Chen, T. Delmas, L. Englert, S. W. Epp, B. Erk, L. Foucar, R. Hartmann, A. Hexemer, M. Huth, J. Kwok, S. R. Leone, J. H. S. Ma, F. R. N. C. Maia, E. Malmerberg, S. Marchesini, D. M. Neumark, B. Poon, J. Prell, D. Rolles, B. Rudek, A. Rudenko, M. Seifrid, K. R. Siefermann, F. P. Sturm, M. Swiggers, J. Ullrich, F. Weise, P. Zwart, C. Bostedt, O. Gessner, and A. F. Vilesov, Science 345, 906 (2014).

${ }^{83}$ C. F. Jones, C. Bernando, S. Erukala, and A. F. Vilesov, J. Phys. Chem. A 123, 5859 (2019).

${ }^{84}$ B. L. Hammond, W. A. Lester, Jr, and P. J. Reynolds, Monte Carlo Methods in ab initio Quantum Chemistry, World Scientific Lecture and Course Notes in Chemistry, Vol. 1 (World Scientific, Singapore, 1975).

${ }^{85}$ D. Blume, M. Lewerenz, F. Huisken, and M. Kaloudis, J. Chem. Phys. 105, 8666 (1996). ${ }^{86}$ M. Lewerenz, J. Chem. Phys. 106, 4596 (1997). 
TABLE I. Parameters of $V_{\mathrm{He}-\mathrm{He}}^{\mathrm{eff}}(R)$ for the cutoff radius $R_{\text {cut }}=1.6 \AA$. The standard deviation between the fitted curve and the original data points for the regions of positive and negative energies are denoted $\mathrm{SD}^{+}$and $\mathrm{SD}^{-}$, respectively.

\begin{tabular}{lc} 
Parameters & Values \\
\hline$R_{e}(\AA)$ & 4.42712 \\
$A\left(\mathrm{~cm}^{-1}\right)$ & 1981.27 \\
$\alpha_{1}$ & -7.86239 \\
$\alpha_{2}$ & 17.1145 \\
$c_{6}\left(\mathrm{~cm}^{-1}\right)$ & -2.34448 \\
$c_{8}\left(\mathrm{~cm}^{-1}\right)$ & 7.45859 \\
$c_{10}\left(\mathrm{~cm}^{-1}\right)$ & -14.879 \\
$c_{12}\left(\mathrm{~cm}^{-1}\right)$ & 8.51457 \\
$a\left(\AA^{-1}\right)$ & 18.347 \\
$b(\AA)$ & 3.6356 \\
\hline $\mathrm{SD}^{-}\left(\mathrm{cm}^{-1}\right)$ & $2.5 \times 10^{-3}$ \\
$\mathrm{SD}^{+}\left(\mathrm{cm}^{-1}\right)$ & 0.71 \\
\hline
\end{tabular}




\section{LIST OF FIGURES}

1 Diatomic interaction potentials used in our model. a) Ar-Ar and He-Ar interaction potentials; b) Average He-Ar ${ }^{+}$interaction potential given by Eq.(7); c) Convergence of the effective He-He interaction potential as a function of the iteration number of the ZPAD method (see text) for $R_{\text {cut }}=1.6 \AA$. Convergence is assumed to be reached when the energy minima of two successive

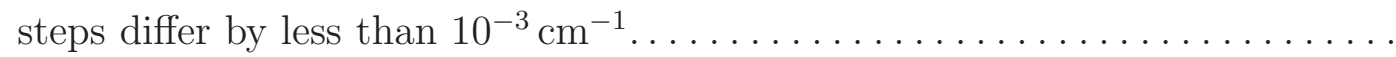

2 Average electronic relaxation time of $\mathrm{Ar}_{4}^{+}$to its ground electronic state as a function of the initial $\mathrm{Ar}_{4}^{+}$electronic state. Electronic states are labeled in order of increasing energy. The average DIM energies of $\mathrm{Ar}_{4}^{+}$(in eV), obtained from the diagonalization of the DIM matrix at $t=0$, are depicted in the inset. The $\mathrm{Ar}^{+}\left({ }^{2} \mathrm{P}\right)+3 \mathrm{Ar}$ total dissociation threshold is the reference for energies. .

3 Theoretical mass spectra of $\mathrm{Ar}_{4}^{+} \mathrm{He}_{1000}$ at the end of the 100 ps nonadiabatic simulation (plot a) and at the end of the overall 500 ps simulation (plot b). $\mathrm{Ar}_{2}^{+} \mathrm{He}_{q}$ and $\mathrm{Ar}_{3}^{+} \mathrm{He}_{q}$ fragment progressions for masses below 160 amu are reported as insets. When two peaks are nearly superimposed their intensities are summed, the corresponding peak is put at the average mass, and a label " $\times 2$ " is added on top of this peak. Sum peaks occur for the pairs $\left(\mathrm{Ar}_{3}^{+}\right.$, $\left.\mathrm{Ar}_{2}^{+} \mathrm{He}_{10}\right),\left(\mathrm{Ar}_{3}^{+} \mathrm{He}, \mathrm{Ar}_{2}^{+} \mathrm{He}_{11}\right),\left(\mathrm{Ar}_{3}^{+} \mathrm{He}_{3}, \mathrm{Ar}_{2}^{+} \mathrm{He}_{13}\right)$, and $\left(\mathrm{Ar}_{3}^{+} \mathrm{He}_{4}, \mathrm{Ar}_{2}^{+} \mathrm{He}_{14}\right)$. The relative abundances of the fragments belonging to these four pairs are as follows:

Inset of plot a: $(25 \%, 75 \%),(50 \%, 50 \%),(75 \%, 25 \%)$, and $(50 \%, 50 \%)$.

Inset of plot b: $(83 \%, 17 \%),(67 \%, 33 \%)$, and $(80 \%, 20 \%) \ldots \ldots \ldots \ldots$

4 Relative abundances (in \%) of $\operatorname{Ar}_{n}^{+} \mathrm{He}_{q}(2 \leq n \leq 4,0 \leq q \leq 1000)$ fragments at the end of the dynamics. The histogram boxes correspond to four groups of ionic fragments, namely bare ions: $q=0$ (label B); small ions: $0<q \leq 50$ (label S); medium-sized ions: $50<q \leq 500$ (label M); large ions: $500<q \leq$ 1000 (label L). . . . . . . . . . . . . . . . . . . . . . . . . .

5 Average Ar-Ar distance within a) $\mathrm{Ar}_{2}^{+}$, b) $\mathrm{Ar}_{3}^{+}$, and c) $\mathrm{Ar}_{4}^{+}$dopants in large $\mathrm{Ar}_{n}^{+} \mathrm{He}_{q}$ clusters $(2 \leq n \leq 4, q>500)$ at $t=500$ ps. $\ldots \ldots \ldots \ldots \ldots \ldots$ 
6 Radial distributions of Ar-He distances between argon atoms within $\mathrm{Ar}_{2}^{+}$or $\mathrm{Ar}_{3}^{+}$cores (black and red lines, respectively) or isolated neutral argon atoms (blue line) and surrounding helium atoms in large $\operatorname{Ar}_{n}^{+} \mathrm{He}_{q}$ fragments $(2 \leq$ $n \leq 4, q>500)$ at $t=500 \mathrm{ps}$.

7 Time evolution of relative abundances (in \%) of $\mathrm{Ar}_{n}^{+} \mathrm{He}_{q}(2 \leq n \leq 4, q \leq 1000$ ) fragments upon electron impact ionization of $\mathrm{Ar}_{4}$ inside a ${ }^{4} \mathrm{He}_{1000}$ droplet. a) Large fragments (or remaining droplets, $q>500$ ) containing an $\mathrm{Ar}_{4}^{+}$ion; b) Large ( $q>500$, full lines) or intermediate-sized (50<q $\leq 500$, dashed lines) ionic fragments for $n=2$ (blue) and $n=3$ (red); c) Small ionic fragments $(0<q \leq 50$, dashed lines) or bare ions ( $q=0$, full lines) for $n=2$ (blue) and $n=3$ (red). The green lines are linear or exponential fits (see text). In order to avoid congestion, $\mathrm{Ar}_{4}^{+} \mathrm{He}_{q}$ fragments with less than 500 helium atoms were not plotted because of their negligible abundances $(<0.7 \%) \ldots \ldots \ldots \ldots$.

8 Selected snapshots of trajectories showing the fast ejection of $\mathrm{Ar}_{2}^{+}$upon $\mathrm{Ar}_{4}$ ionization to the $\mathrm{Ar}_{4}^{+}$electronic state of highest energy (state 12, $V_{12} \approx$ $0.141 \mathrm{eV}$ ), and the trapping of $\mathrm{Ar}_{2}^{+}$upon $\mathrm{Ar}_{4}$ ionization to the $\mathrm{Ar}_{4}^{+}$electronic state of lowest energy (state $1, V_{1} \approx-0.478 \mathrm{eV}$ ). The color scale on the left aims to provide a visual estimate of charge $q_{i}^{(j)}$ carried by the four argon atoms, from purple for Ar to red for $\mathrm{Ar}^{+}$.

$9 \quad$ Selected snapshots of trajectories showing geminate recombinations yielding a secondary fragmentation event and the release of $\mathrm{Ar}_{3}^{+}$in the gas phase for an initial ionization in the $\mathrm{Ar}_{4}^{+}$electronic state $6\left(V_{6} \approx-0.209 \mathrm{eV}\right)$. The color scale on the left aims to provide a visual estimate of charge $q_{i}^{(j)}$ carried by the four argon atoms, from purple for Ar to red for $\mathrm{Ar}^{+}$ 
10 a) Percentage of trajectories subject to fast ejection of small ionic fragments (red lines), long-term trapping of $\operatorname{Ar}_{n}^{+}$( $2 \leq n \leq 4$, blue lines), or intermediate processes (black lines) as a function of the initial $\mathrm{Ar}_{4}^{+}$electronic state number, these states being sorted in ascending order of energy. The value of $100 \%$ is obtained by summing the percentages of the three curves and twelve electronic states. b) Point-cloud diagram showing the mass of ionic fragments containing $\mathrm{Ar}_{2}^{+}$(circles), $\mathrm{Ar}_{3}^{+}$(squares), and $\mathrm{Ar}_{4}^{+}$(triangles) as a function of the initial $\mathrm{Ar}_{4}^{+}$electronic state number at $t=500 \mathrm{ps}$. The color of symbols refers to the dynamical process involved to produce these fragments ( red $=$ fast ejection, blue $=$ long-term trapping, black $=$ intermediate process $).$

11 Typical kinetic energy distribution of helium atoms. The intensity of the distribution is a percentage of the total number of ejected helium atoms. .... 

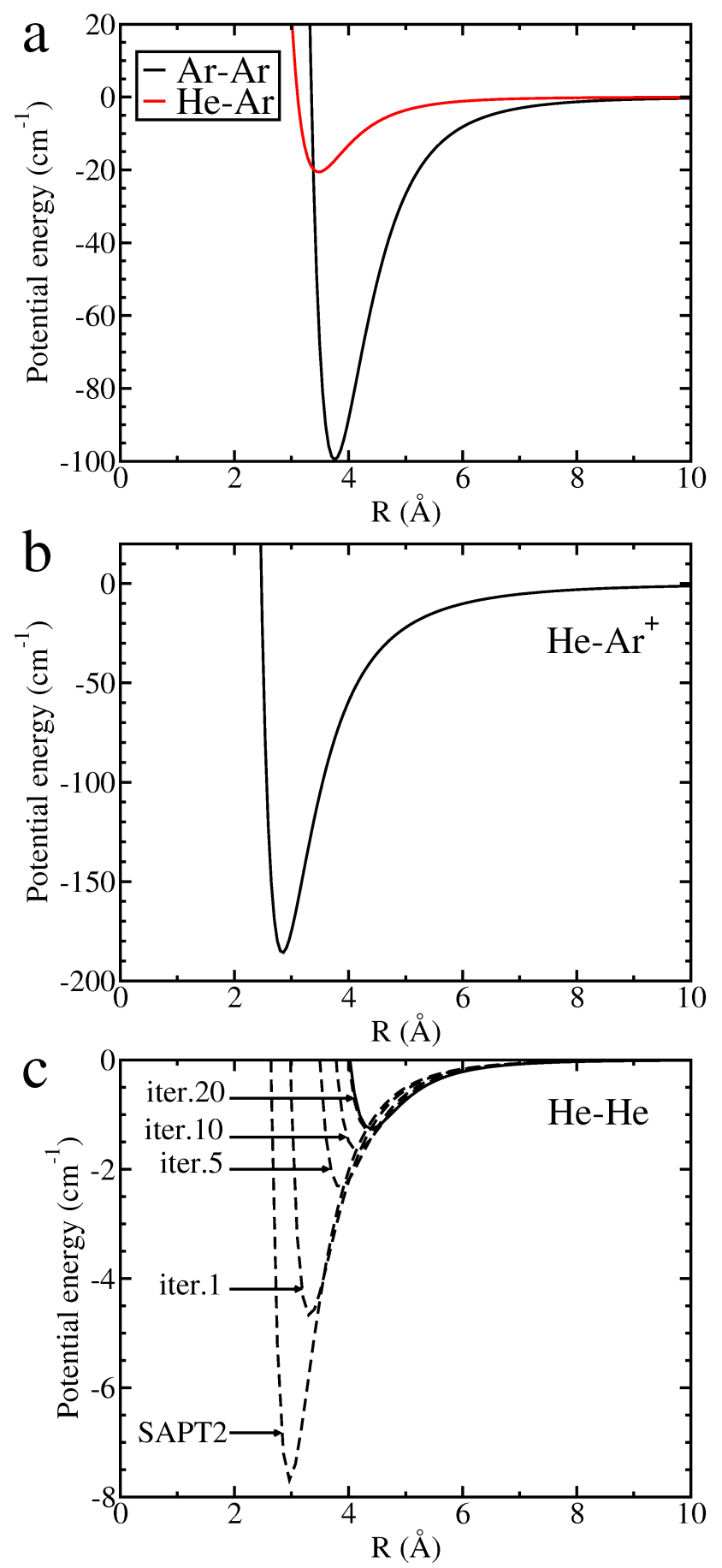

FIG. 1. Diatomic interaction potentials used in our model. a) Ar-Ar and He-Ar interaction potentials; b) Average He-Ar ${ }^{+}$interaction potential given by Eq.(7); c) Convergence of the effective He-He interaction potential as a function of the iteration number of the ZPAD method (see text) for $R_{\text {cut }}=1.6 \AA$. Convergence is assumed to be reached when the energy minima of two successive steps differ by less than $10^{-3} \mathrm{~cm}^{-1}$. 


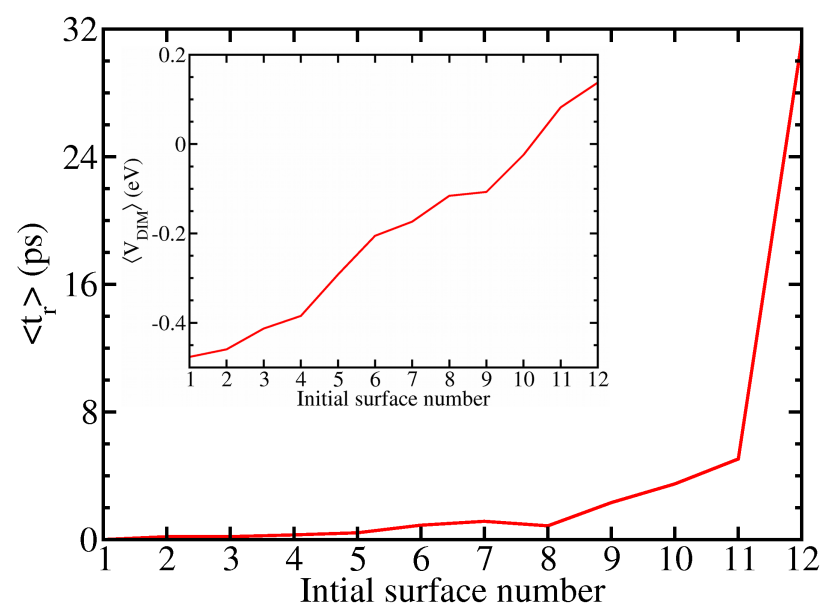

FIG. 2. Average electronic relaxation time of $\mathrm{Ar}_{4}^{+}$to its ground electronic state as a function of the initial $\mathrm{Ar}_{4}^{+}$electronic state. Electronic states are labeled in order of increasing energy. The average DIM energies of $\mathrm{Ar}_{4}^{+}$(in eV), obtained from the diagonalization of the DIM matrix at $t=0$, are depicted in the inset. $\operatorname{The~} \mathrm{Ar}^{+}\left({ }^{2} \mathrm{P}\right)+3 \mathrm{Ar}$ total dissociation threshold is the reference for energies. 

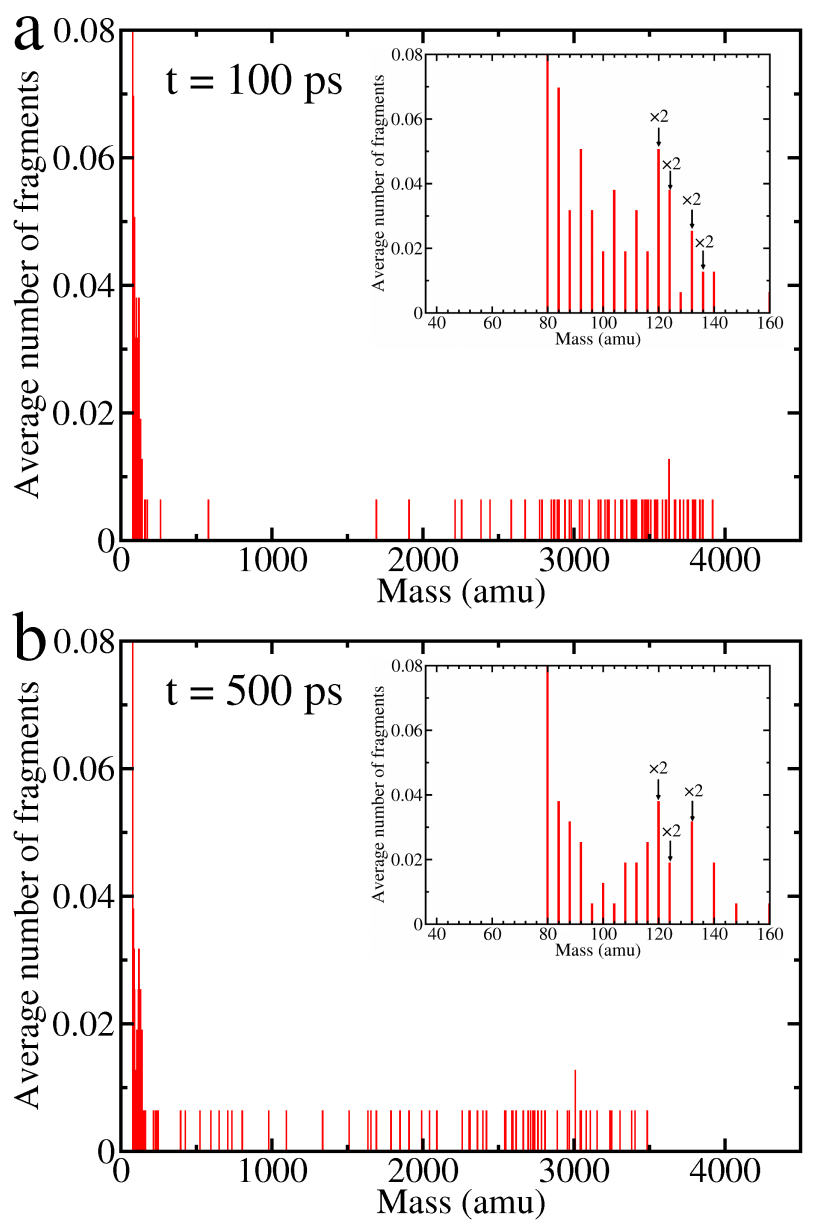

FIG. 3. Theoretical mass spectra of $\mathrm{Ar}_{4}^{+} \mathrm{He}_{1000}$ at the end of the 100 ps nonadiabatic simulation (plot a) and at the end of the overall 500 ps simulation (plot b). $\mathrm{Ar}_{2}^{+} \mathrm{He}_{q}$ and $\mathrm{Ar}_{3}^{+} \mathrm{He}_{q}$ fragment progressions for masses below 160 amu are reported as insets. When two peaks are nearly superimposed their intensities are summed, the corresponding peak is put at the average mass, and a label " $\times 2$ " is added on top of this peak. Sum peaks occur for the pairs $\left(\mathrm{Ar}_{3}^{+}, \mathrm{Ar}_{2}^{+} \mathrm{He}_{10}\right),\left(\mathrm{Ar}_{3}^{+} \mathrm{He}_{\text {, }}\right.$ $\left.\mathrm{Ar}_{2}^{+} \mathrm{He}_{11}\right),\left(\mathrm{Ar}_{3}^{+} \mathrm{He}_{3}, \mathrm{Ar}_{2}^{+} \mathrm{He}_{13}\right)$, and $\left(\mathrm{Ar}_{3}^{+} \mathrm{He}_{4}, \mathrm{Ar}_{2}^{+} \mathrm{He}_{14}\right)$. The relative abundances of the fragments belonging to these four pairs are as follows:

Inset of plot a: $(25 \%, 75 \%),(50 \%, 50 \%),(75 \%, 25 \%)$, and $(50 \%, 50 \%)$.

Inset of plot b: $(83 \%, 17 \%),(67 \%, 33 \%)$, and $(80 \%, 20 \%)$. 


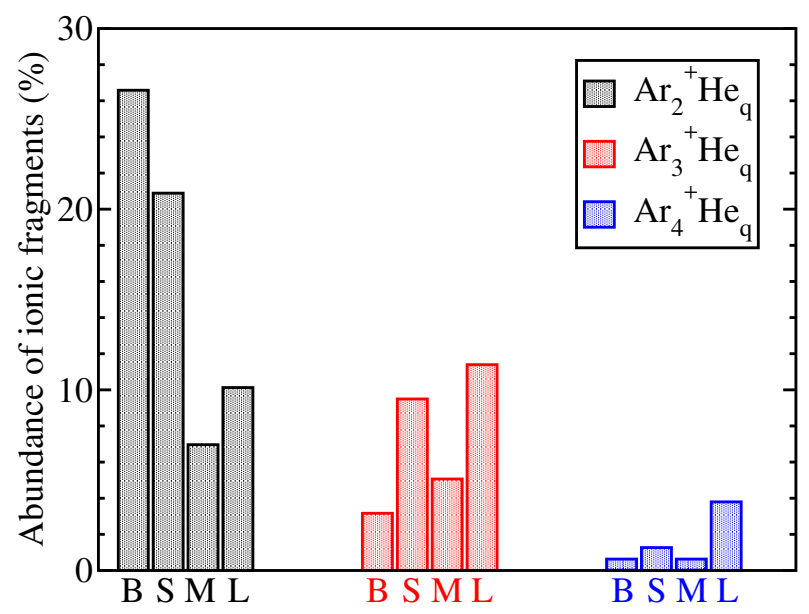

FIG. 4. Relative abundances (in \%) of $\operatorname{Ar}_{n}^{+} \operatorname{He}_{q}(2 \leq n \leq 4,0 \leq q \leq 1000)$ fragments at the end of the dynamics. The histogram boxes correspond to four groups of ionic fragments, namely bare ions: $q=0$ (label B); small ions: $0<q \leq 50$ (label S); medium-sized ions: $50<q \leq 500$ (label M); large ions: $500<q \leq 1000$ (label L). 

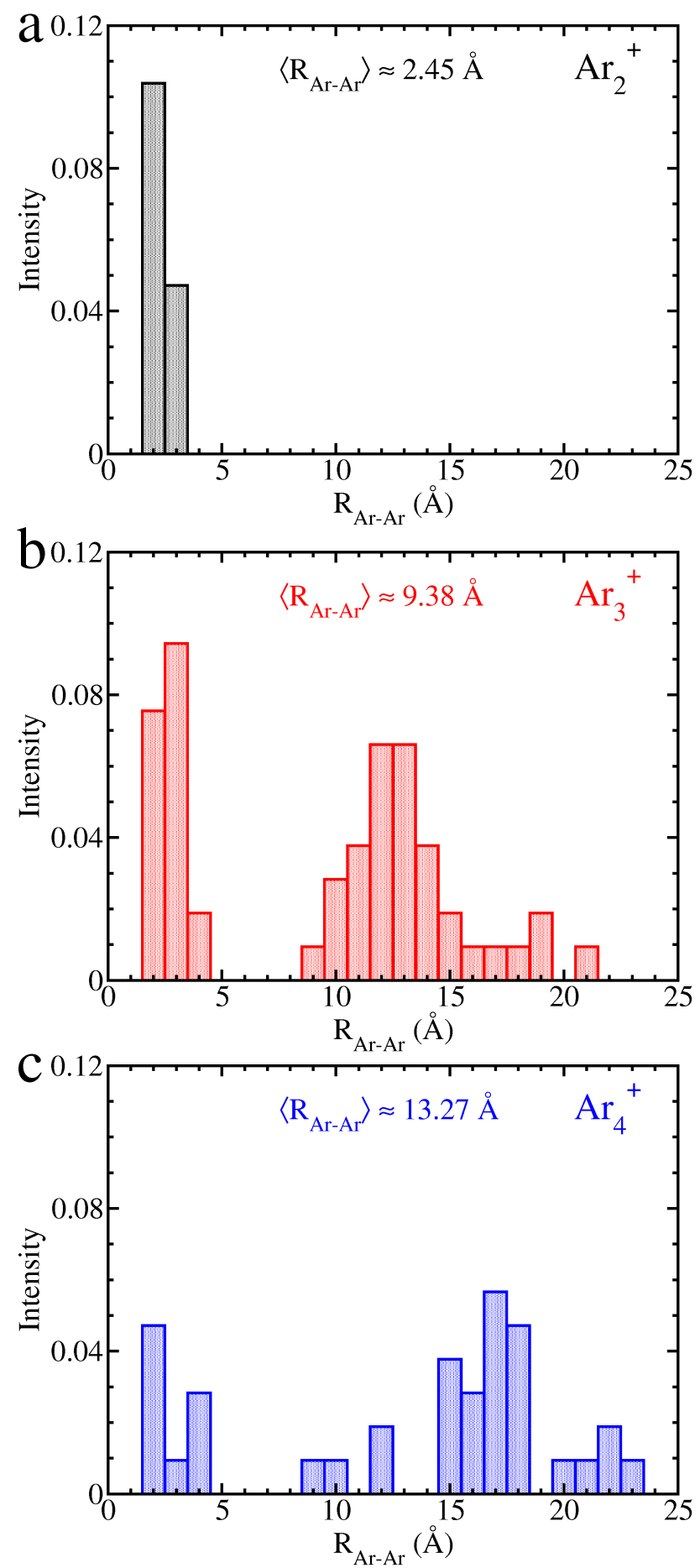

FIG. 5. Average Ar-Ar distance within a) $\mathrm{Ar}_{2}^{+}$, b) $\mathrm{Ar}_{3}^{+}$, and c) $\mathrm{Ar}_{4}^{+}$dopants in large $\mathrm{Ar}_{n}^{+} \mathrm{He}_{q}$ clusters $(2 \leq n \leq 4, q>500)$ at $t=500$ ps. 


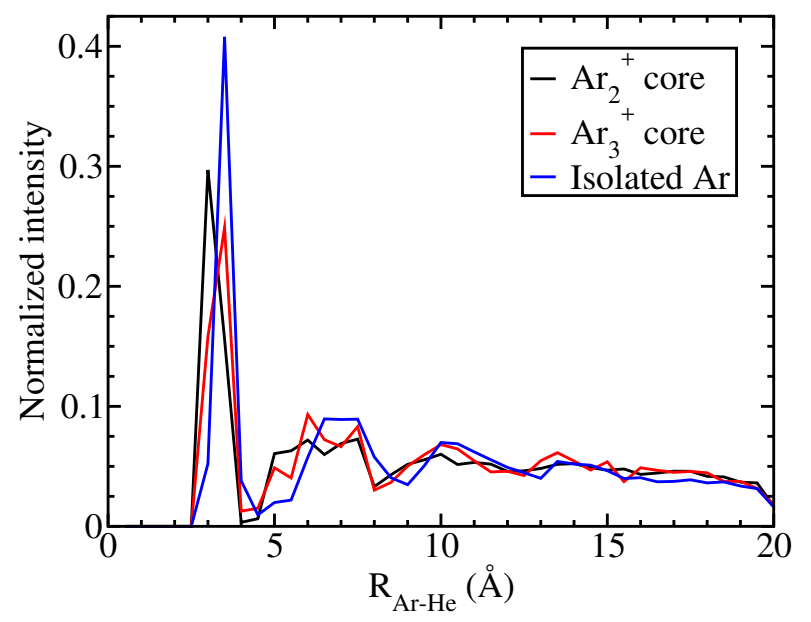

FIG. 6. Radial distributions of Ar-He distances between argon atoms within $\mathrm{Ar}_{2}^{+}$or $\mathrm{Ar}_{3}^{+}$cores (black and red lines, respectively) or isolated neutral argon atoms (blue line) and surrounding helium atoms in large $\mathrm{Ar}_{n}^{+} \mathrm{He}_{q}$ fragments $(2 \leq n \leq 4, q>500)$ at $t=500 \mathrm{ps}$. 

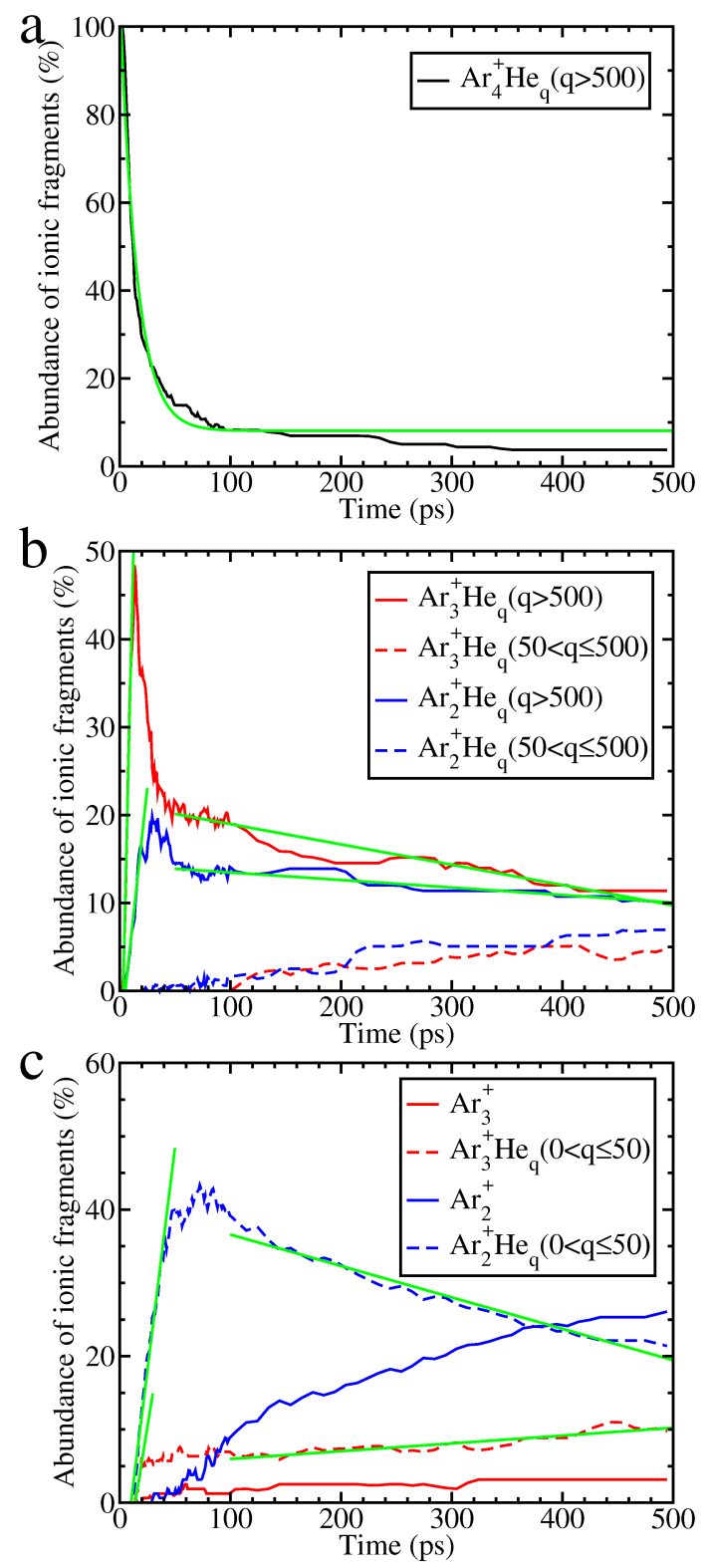

FIG. 7. Time evolution of relative abundances (in \%) of $\operatorname{Ar}_{n}^{+} \mathrm{He}_{q}(2 \leq n \leq 4, q \leq 1000)$ fragments upon electron impact ionization of $\mathrm{Ar}_{4}$ inside a ${ }^{4} \mathrm{He}_{1000}$ droplet. a) Large fragments (or remaining droplets, $q>500)$ containing an $\operatorname{Ar}_{4}^{+}$ion; b) Large $(q>500$, full lines $)$ or intermediate-sized (50<q $\leq 500$, dashed lines) ionic fragments for $n=2$ (blue) and $n=3$ (red); c) Small ionic fragments $(0<q \leq 50$, dashed lines) or bare ions ( $q=0$, full lines) for $n=2$ (blue) and $n=3$ (red). The green lines are linear or exponential fits (see text). In order to avoid congestion, $\mathrm{Ar}_{4}^{+} \mathrm{He}_{q}$ fragments with less than 500 helium atoms were not plotted because of their negligible abundances $(<0.7 \%)$. 


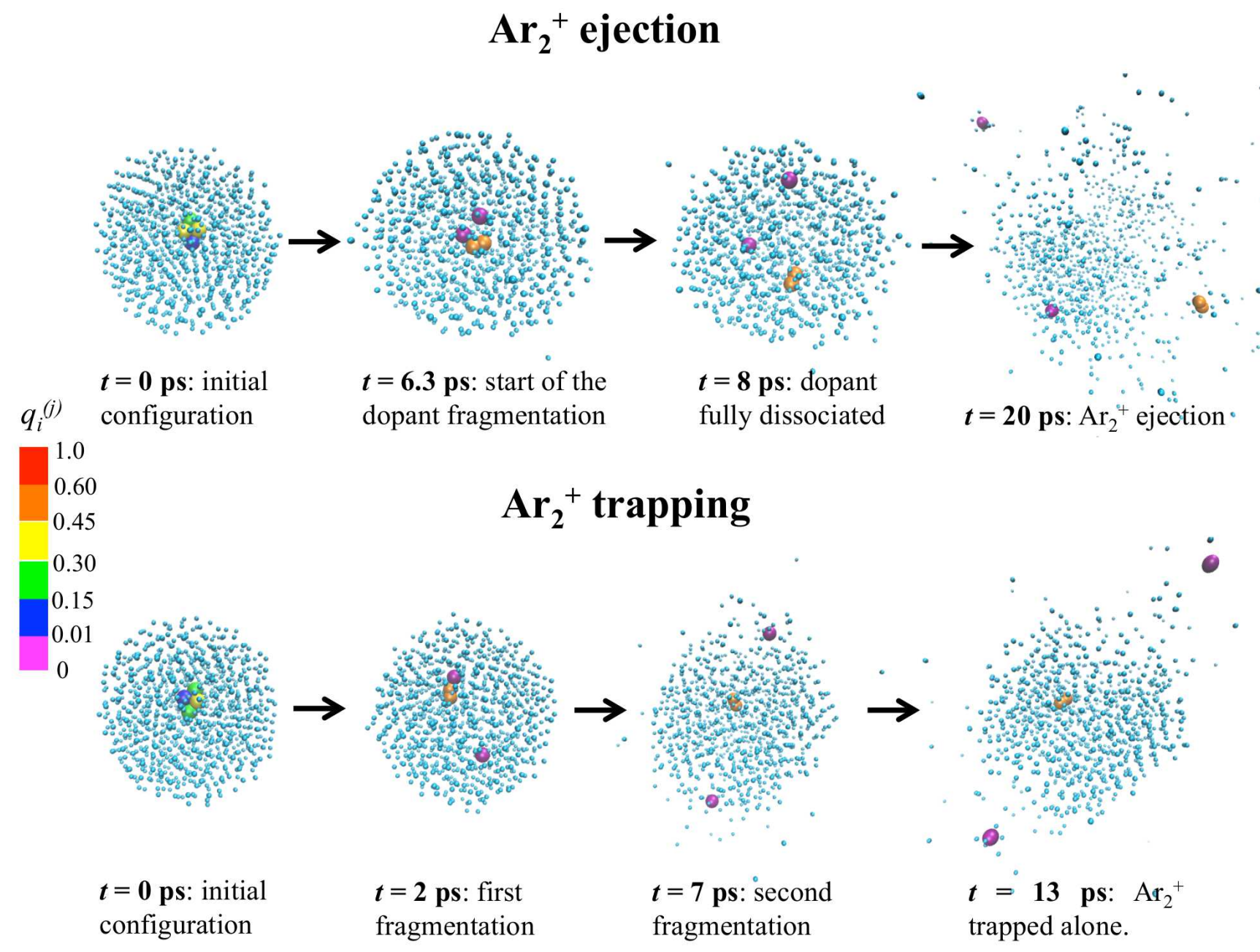

FIG. 8. Selected snapshots of trajectories showing the fast ejection of $\mathrm{Ar}_{2}^{+}$upon $\mathrm{Ar}_{4}$ ionization to the $\mathrm{Ar}_{4}^{+}$electronic state of highest energy (state $12, V_{12} \approx 0.141 \mathrm{eV}$ ), and the trapping of $\mathrm{Ar}_{2}^{+}$ upon $\mathrm{Ar}_{4}$ ionization to the $\mathrm{Ar}_{4}^{+}$electronic state of lowest energy (state $1, V_{1} \approx-0.478 \mathrm{eV}$ ). The color scale on the left aims to provide a visual estimate of charge $q_{i}^{(j)}$ carried by the four argon atoms, from purple for Ar to red for $\mathrm{Ar}^{+}$. 


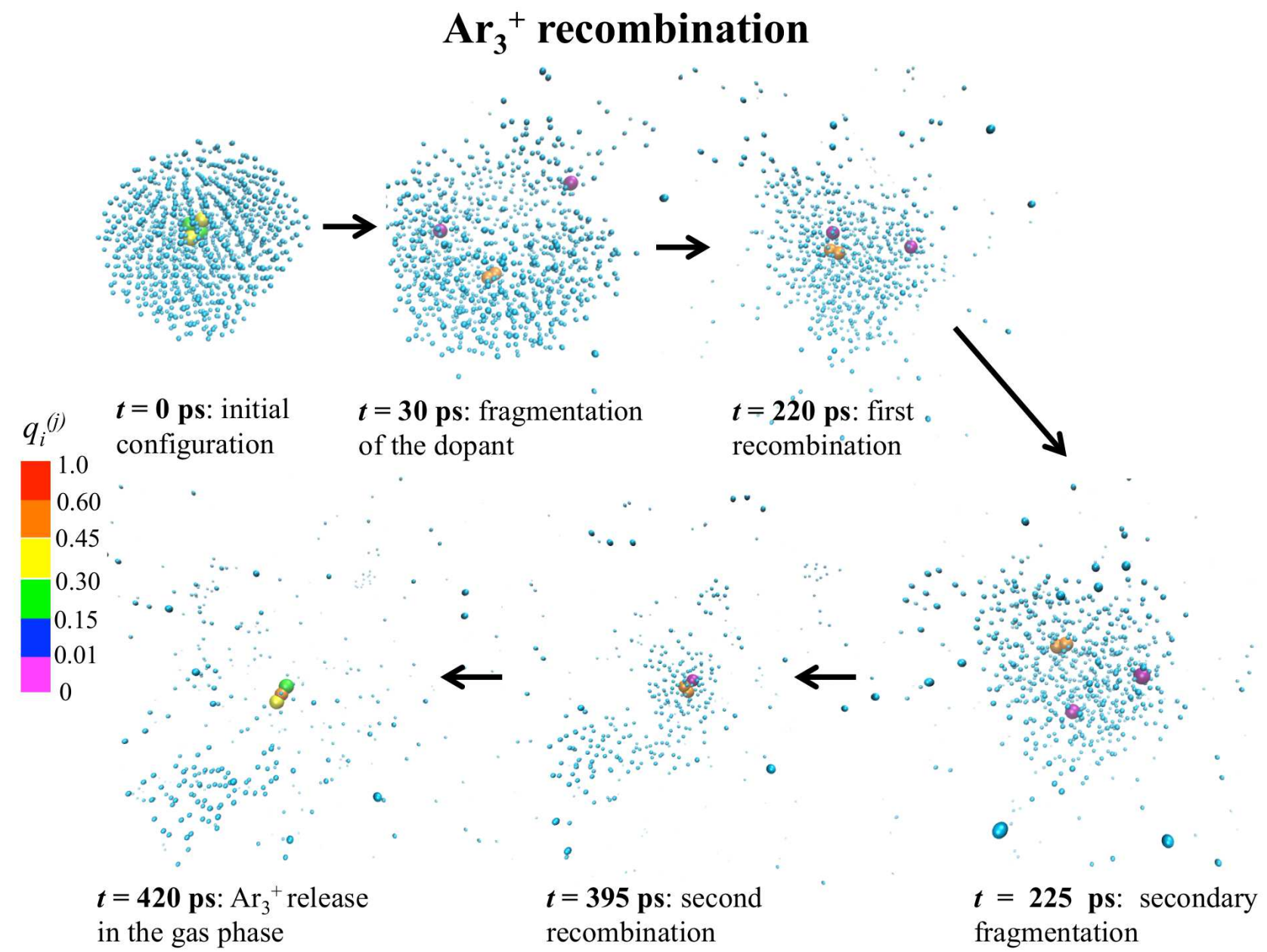

FIG. 9. Selected snapshots of trajectories showing geminate recombinations yielding a secondary fragmentation event and the release of $\mathrm{Ar}_{3}^{+}$in the gas phase for an initial ionization in the $\mathrm{Ar}_{4}^{+}$ electronic state $6\left(V_{6} \approx-0.209 \mathrm{eV}\right)$. The color scale on the left aims to provide a visual estimate of charge $q_{i}^{(j)}$ carried by the four argon atoms, from purple for Ar to red for $\mathrm{Ar}^{+}$. 

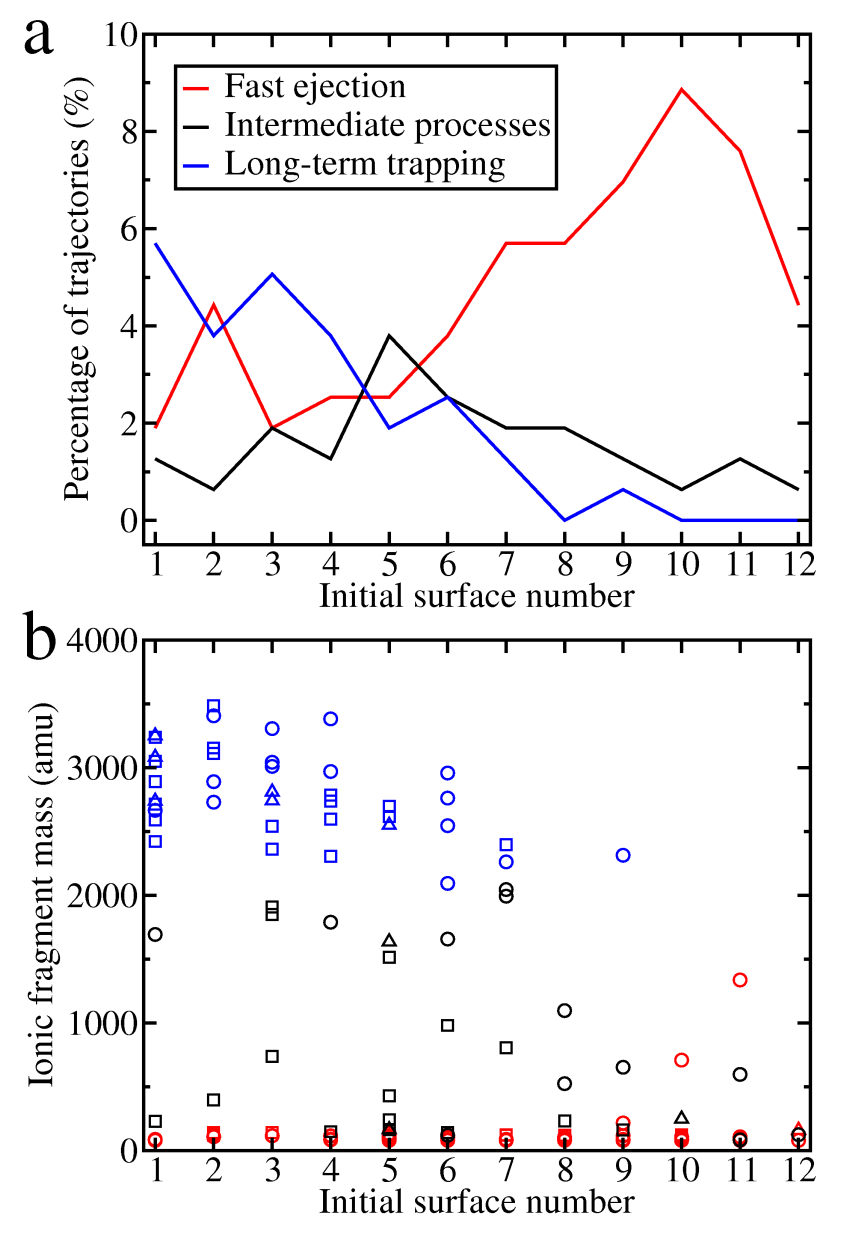

FIG. 10. a) Percentage of trajectories subject to fast ejection of small ionic fragments (red lines), long-term trapping of $\operatorname{Ar}_{n}^{+}(2 \leq n \leq 4$, blue lines), or intermediate processes (black lines) as a function of the initial $\mathrm{Ar}_{4}^{+}$electronic state number, these states being sorted in ascending order of energy. The value of $100 \%$ is obtained by summing the percentages of the three curves and twelve electronic states. b) Point-cloud diagram showing the mass of ionic fragments containing $\mathrm{Ar}_{2}^{+}$(circles), $\mathrm{Ar}_{3}^{+}$(squares), and $\mathrm{Ar}_{4}^{+}$(triangles) as a function of the initial $\mathrm{Ar}_{4}^{+}$electronic state number at $t=500 \mathrm{ps}$. The color of symbols refers to the dynamical process involved to produce these fragments $(\mathrm{red}=$ fast ejection, blue $=$ long-term trapping, black = intermediate process). 


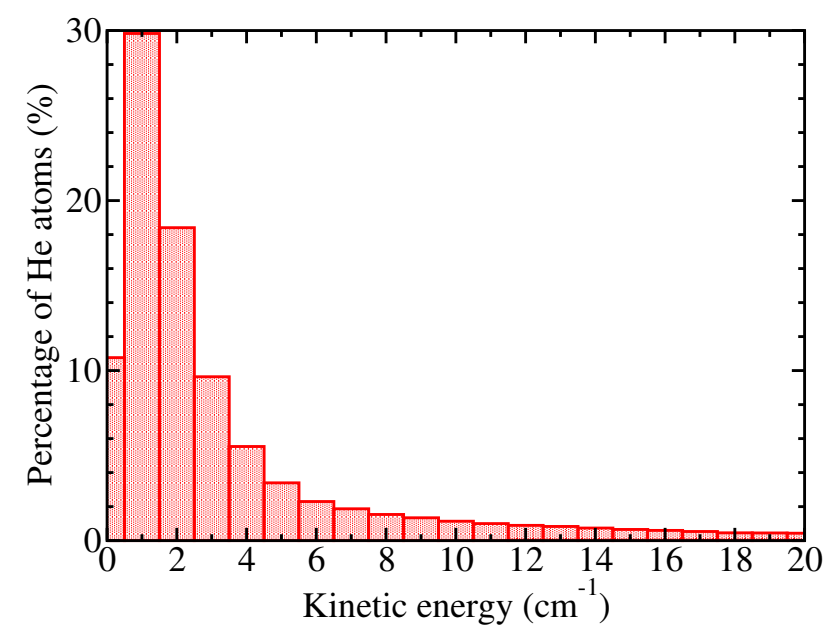

FIG. 11. Typical kinetic energy distribution of helium atoms. The intensity of the distribution is a percentage of the total number of ejected helium atoms. 